Fine- and hyperfine-structure effects in molecular photoionization. II. Resonanceenhanced multiphoton ionization and hyperfine-selective generation of molecular cations

Matthias Germann and Stefan Willitsch

Citation: The Journal of Chemical Physics 145, 044315 (2016); doi: 10.1063/1.4955303

View online: http://dx.doi.org/10.1063/1.4955303

View Table of Contents: http://aip.scitation.org/toc/jcp/145/4

Published by the American Institute of Physics

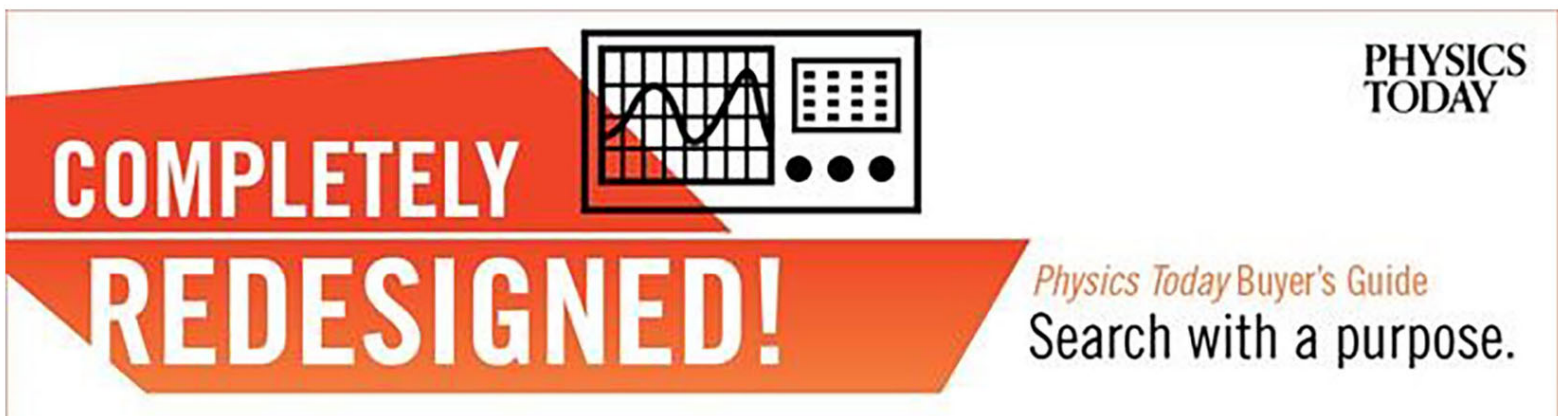




\title{
Fine- and hyperfine-structure effects in molecular photoionization. II. Resonance-enhanced multiphoton ionization and hyperfine-selective generation of molecular cations
}

\author{
Matthias Germann and Stefan Willitsch ${ }^{\text {a) }}$ \\ Department of Chemistry, University of Basel, Klingelbergstrasse 80, 4056 Basel, Switzerland
}

(Received 2 June 2016; accepted 23 June 2016; published online 27 July 2016)

\begin{abstract}
Resonance-enhanced multiphoton ionization (REMPI) is a widely used technique for studying molecular photoionization and producing molecular cations for spectroscopy and dynamics studies. Here, we present a model for describing hyperfine-structure effects in the REMPI process and for predicting hyperfine populations in molecular ions produced by this method. This model is a generalization of our model for fine- and hyperfine-structure effects in one-photon ionization of molecules presented in Paper I [M. Germann and S. Willitsch, J. Chem. Phys. 145, 044314 (2016)]. This generalization is achieved by covering two main aspects: (1) treatment of the neutral bound-bound transition including the hyperfine structure that makes up the first step of the REMPI process and (2) modification of our ionization model to account for anisotropic populations resulting from this first excitation step. Our findings may be used for analyzing results from experiments with molecular ions produced by REMPI and may serve as a theoretical background for hyperfine-selective ionization experiments. Published by AIP Publishing. [http://dx.doi.org/10.1063/1.4955303]
\end{abstract}

\section{INTRODUCTION}

Resonance-enhanced multiphoton ionization (REMPI) ionization of atoms or molecules by several photons via resonant excitation of the neutral precursor specieshas become a well-established method to study the photoionization of molecules ${ }^{1,2}$ and produce molecular cations for dynamics and spectroscopy experiments ${ }^{3-6}$ over the last decades. Multiphoton ionization avoids the need for vacuumultraviolet radiation, while resonant ionization, i.e., ionization via an excited state of the neutral precursor molecule, can increase the photoionization yield and improves the selectivity of the ionization process. In particular, selection and propensity rules governing the REMPI process may be exploited for rotational-vibrational state-selective production of molecular cations. ${ }^{3,4,7-9}$

In the preceding article, ${ }^{10}$ cited as Paper I below, we have presented a model for fine-structure-(fs) and hyperfinestructure-(hfs)-resolved photoionization intensities in direct, one-photon ionization of molecules. Here, we extend our model to cover two-color multiphoton ionization.

We focus on the $\left[2+1^{\prime}\right]$ REMPI scheme, i.e., excitation by a two-photon transition from the neutral-ground to a neutral-excited state followed by one-photon ionization of the neutral excited state. The method presented, however, is general and may be extended to other two-color REMPI schemes such as $\left[1+1^{\prime}\right]$.

\section{GENERAL CONSIDERATIONS}

The scheme of a $\left[2+1^{\prime}\right]$ REMPI process is illustrated in Fig. 1: A neutral diatomic molecule $\mathrm{AB}$ is first excited

\footnotetext{
a)Electronic mail: stefan.willitsch@unibas.ch
}

from the electronic ground state to a neutral electronically or vibrationally excited state $\left(\mathrm{AB}^{*}\right)$ by absorption of two photons at the (angular) frequency $\omega_{1}$. Thereafter, the molecule is ionized by absorption of a third photon at a different frequency $\omega_{2}$ forming the molecular ion $\mathrm{AB}^{+}$. Following this picture, we describe the $\left[2+1^{\prime}\right]$ REMPI process as a sequence of two independent steps: a transition from the neutral electronicground-state molecule $\mathrm{AB}$ to the neutral excited molecule $\mathrm{AB}^{*}$ and a subsequent ionization of this molecule yielding the molecular ion $\mathrm{AB}^{+}$.

For the excitation step $\left(\mathrm{AB} \rightarrow \mathrm{AB}^{*}\right)$, we will develop a model for hfs-resolved two-photon transitions between bound states. Using this model, we calculate the excitation rate $R\left(\mathrm{AB} \rightarrow \mathrm{AB}^{*}\right)$ and hence the relative rotational and hyperfine populations $\rho^{\prime}$ of excited molecules $\mathrm{AB}^{*}$. The ionization of the excited molecules is then described by our ionization model developed in Paper $\mathrm{I},{ }^{10}$ with the excited state population $\rho^{\prime}$ used in lieu of the thermal ground state population.

The excitation of the neutral molecules $\mathrm{AB}$ to $\mathrm{AB}^{*}$ by polarized radiation leads to an anisotropic population $\rho^{\prime}$, meaning that the several Zeeman states in the excited state are unequally populated. ${ }^{11,12}$ Since for our photoionization model presented in Paper $\mathrm{I}^{10}{ }^{10}$ isotropic populations have been implicitly assumed, we need to adapt that model for anisotropic excited populations of the neutral in REMPI.

Our manuscript is structured as follows: In Sec. III, we discuss the excitation step, i.e., we develop a model for hyperfine structure effects in the initial two-photon excitation transition. Besides the application in our $\left[2+1^{\prime}\right]$ REMPI model, the theory developed in that section is also generally applicable to hyperfine-structure-resolved two-photon boundbound transitions.

In Sec. IV, we discuss the ionization step, i.e., we develop the above-mentioned adaptations of our ionization model from 


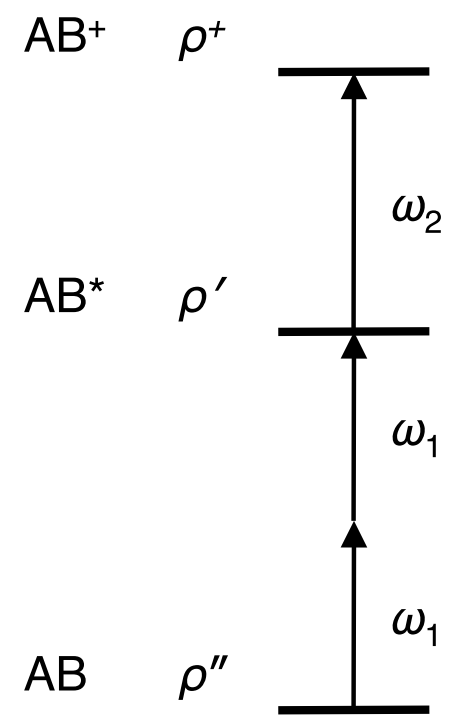

FIG. 1. Schematic of a $\left[2+1^{\prime}\right]$ resonance-enhanced multiphoton ionization process: neutral molecules $\mathrm{AB}$ are excited by absorption of two photons at an angular frequency $\omega_{1}$ yielding excited molecules $\mathrm{AB}^{*}$. These are then ionized by absorption of a third photon at a different frequency $\omega_{2}$, resulting in the generation of molecular ions $\mathrm{AB}^{+}$. The populations associated with $\mathrm{AB}, \mathrm{AB}^{*}$, and $\mathrm{AB}^{+}$are denoted by $\rho^{\prime \prime}, \rho^{\prime}$, and $\rho^{+}$, respectively.

Paper $\mathrm{I}^{10}$ for ionization of an anisotropically populated neutral state.

In Sec. V, we then combine these results to a complete model for the $\left[2+1^{\prime}\right]$ REMPI process. Moreover, we shortly indicate how to adapt our model to other REMPI processes besides the $\left[2+1^{\prime}\right]$ scheme discussed here.

The implications of our model are shown in Sec. VI using the $\left[2+1^{\prime}\right]$ REMPI of $\mathrm{N}_{2}$ via the neutral excited $\mathrm{a}^{\prime \prime}{ }^{1} \Sigma_{\mathrm{g}}^{+}$state as a representative example.

Finally, we summarize our findings in Sec. VII.

\section{EXCITATION STEP: HFS-RESOLVED NON-RESONANT TWO-PHOTON TRANSITIONS}

Two- and multiphoton transitions in diatomic molecules have been discussed in several previous publications, e.g., by Bray and Hochstrasser, ${ }^{13}$ Maïnos, ${ }^{14}$ Dixit and McKoy, ${ }^{15}$ Lefebvre-Brion and Field ${ }^{16}$ as well as Hippler. ${ }^{17}$ These treat the "Göppert-Meyer mechanism" first described in Refs. 18 and 19 . Here, we will extend these treatments to hyperfinestructure-resolved transitions.

The transition rate $R_{\mathrm{g} \rightarrow \mathrm{e}}$ for the excitation of the molecule from the ground state $|\mathrm{g}\rangle$ to the excited state $|\mathrm{e}\rangle$ is expressed as a product of the radiation intensity $I_{0}$ and the two-photon line strength $S_{\mathrm{ge}},{ }^{17}$

$$
R_{\mathrm{g} \rightarrow \mathrm{e}} \propto\left(I_{0}\right)^{2} S_{\mathrm{ge}}
$$

Note that for two-photon transitions, the radiation intensity enters squared in the two-photon transition rate.

According to the Göppert-Meyer mechanism, the ground and excited states are connected by two off-resonant onephoton transitions via intermediate states. As excitation is possible via different intermediate states, all possible virtual transition routes are summed, weighted by the inverse mismatch between the frequency of the photons absorbed and the one-photon transition frequencies to the intermediate states. The two-photon line strength factor $S_{\mathrm{ge}}$ is given therefore as ${ }^{17}$

$$
S_{\mathrm{ge}}=\sum_{M_{\mathrm{e}}, M_{\mathrm{g}}}\left|\sum_{\mathrm{i}} \frac{1}{\omega_{\mathrm{ig}}-\omega_{1}}\left\langle\mathrm{e}\left|\mathbf{e}_{\sigma} \cdot \boldsymbol{\mu}\right| \mathrm{i}\right\rangle\left\langle\mathrm{i}\left|\mathbf{e}_{\sigma} \cdot \boldsymbol{\mu}\right| \mathrm{g}\right\rangle\right|^{2} .
$$

Here, $|i\rangle$ is the intermediate state of the virtual one-photon transition route with the sum over $i$ including all accessible intermediate states. $M_{\mathrm{g}}$ and $M_{\mathrm{e}}$ label the different Zeeman states in the ground and the excited states, respectively. The term $\omega_{\text {ig }}-\omega_{1}$ represents the mismatch between the groundintermediate-state transition energy $\hbar \omega_{\text {ig }}$ and the photon energy $\hbar \omega_{1}$ (see Fig. 2(a)). Moreover, $\boldsymbol{\mu}$ is the electricdipole operator and $\mathbf{e}_{\sigma}$ the unit polarization vector of the radiation with $\sigma=0$ standing for linear, $\sigma= \pm 1$ for circular polarization.

Assuming that the molecular states may be written as a product of an electronic-vibrational state (labeled "ev") and a nuclear-spin-rotational state (labeled "nsr"), the two-photon line strength in Eq. (2) takes the form

$$
\begin{aligned}
S_{\mathrm{ge}}= & \sum_{M_{\mathrm{e}}, M_{\mathrm{g}}} \mid \sum_{\mathrm{i}_{\mathrm{ev}}} \sum_{\mathrm{i}_{\mathrm{nsr}}} \frac{1}{\omega_{\mathrm{i}_{\mathrm{ev}} \mathrm{i}_{\mathrm{n} s r} \mathrm{~g}}-\omega_{1}} \\
& \times\left\langle\mathrm{e}_{\mathrm{ev}}, \mathrm{e}_{\mathrm{nsr}}\left|\mathbf{e}_{\sigma} \cdot \boldsymbol{\mu}\right| \mathrm{i}_{\mathrm{ev}}, \mathrm{i}_{\mathrm{nsr}}\right\rangle \\
& \times\left.\left\langle\mathrm{i}_{\mathrm{ev}}, \mathrm{i}_{\mathrm{nsr}}\left|\mathbf{e}_{\sigma} \cdot \boldsymbol{\mu}\right| \mathrm{g}_{\mathrm{ev}}, \mathrm{g}_{\mathrm{nsr}}\right\rangle\right|^{2} .
\end{aligned}
$$

Here the sum over all intermediate states has been written as a sum over all electronic-vibrational intermediate states $\sum_{\mathrm{i}_{\mathrm{ev}}}$ and all nuclear-spin-rotational intermediate states $\sum_{\mathrm{i}_{\text {nsr }}}$. Accordingly, $\omega_{\text {ig }}$ has been rewritten as $\omega_{\mathrm{i}_{\mathrm{ev}} \mathrm{i}_{\mathrm{nsr}} \mathrm{g}}$.

To evaluate Eq. (3), we express the scalar product $\mathbf{e}_{\sigma} \cdot \boldsymbol{\mu}$ in spherical tensor notation, change to the molecule-fixed frame by the aid of Wigner rotation matrices, and factorize the transition matrix elements into angular and vibronic terms. For the ground-to-intermediate-state transition matrix element we get

$$
\begin{aligned}
& \left\langle\mathrm{e}_{\mathrm{ev}}, \mathrm{e}_{\mathrm{nsr}}\left|\mathbf{e}_{\sigma} \cdot \boldsymbol{\mu}\right| \mathrm{i}_{\mathrm{ev}}, \mathrm{i}_{\mathrm{nsr}}\right\rangle \\
& =\sum_{\tau_{1}=-1}^{1}\left\langle\mathrm{e}_{\mathrm{ev}}, \mathrm{e}_{\mathrm{nsr}}\left|\left[\mathcal{D}_{\sigma \tau_{1}}^{(1)}\right]^{*} \mathrm{~T}_{\tau_{1}}^{1}[\boldsymbol{\mu}]\right| \mathrm{i}_{\mathrm{ev}}, \mathrm{i}_{\mathrm{nsr}}\right\rangle \\
& \quad=\sum_{\tau_{1}=-1}^{1}\left\langle\mathrm{e}_{\mathrm{ev}}\left|\mathrm{T}_{\tau_{1}}^{1}[\boldsymbol{\mu}]\right| \mathrm{i}_{\mathrm{ev}}\right\rangle\left\langle\mathrm{e}_{\mathrm{nsr}}\left|\left[\mathcal{D}_{\sigma \tau_{1}}^{(1)}\right]^{*}\right| \mathrm{i}_{\mathrm{nsr}}\right\rangle .
\end{aligned}
$$

For the intermediate-to-excited-state transition matrix element, an analogous expression is obtained.

Thus, the two-photon line strength is

$$
\begin{aligned}
S_{\mathrm{ge}}= & \sum_{M_{\mathrm{e}}, M_{\mathrm{g}}} \mid \sum_{\mathrm{i}_{\mathrm{ev}}} \sum_{\mathrm{i}_{\mathrm{nsr}}} \frac{1}{\omega_{\mathrm{i}_{\mathrm{ev}} \mathrm{i}_{\mathrm{nsr}} \mathrm{g}}-\omega_{1}} \sum_{\tau_{1}, \tau_{2}}\left\langle\mathrm{e}_{\mathrm{ev}}\left|\mathrm{T}_{\tau_{1}}^{1}[\boldsymbol{\mu}]\right| \mathrm{i}_{\mathrm{ev}}\right\rangle \\
& \times\left\langle\mathrm{i}_{\mathrm{ev}}\left|\mathrm{T}_{\tau_{2}}^{1}[\boldsymbol{\mu}]\right| \mathrm{g}_{\mathrm{ev}}\right\rangle\left\langle\mathrm{e}_{\mathrm{nsr}}\left|\left[\mathcal{D}_{\sigma \tau_{1}}^{(1)}\right]^{*}\right| \mathrm{i}_{\mathrm{nsr}}\right\rangle \\
& \times\left.\left\langle\mathrm{i}_{\mathrm{nsr}}\left|\left[\mathcal{D}_{\sigma \tau_{2}}^{(1)}\right]^{*}\right| \mathrm{g}_{\mathrm{nsr}}\right\rangle\right|^{2} .
\end{aligned}
$$

As indicated by the notation, the energy difference between ground level and intermediate level $\hbar \omega_{\mathrm{i}_{\mathrm{ev}} \mathrm{i}_{\text {nsr }} \mathrm{g}}$ depends in 
(a) le $\rangle$

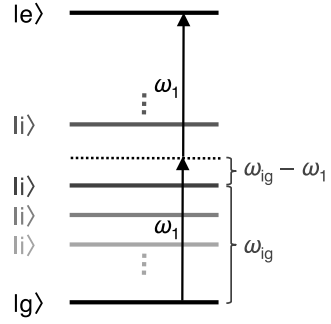

(b)

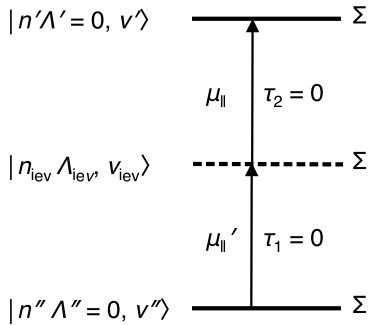

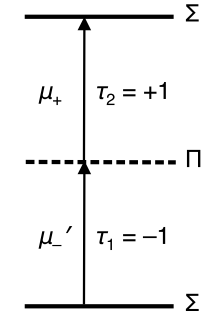

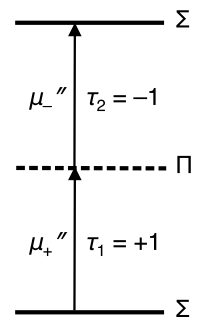

FIG. 2. Mechanism of two-photon transitions: (a) Two-photon transitions are described by virtual one-photon transition routes connecting the ground state $|\mathrm{g}\rangle$ to an excited state $|\mathrm{e}\rangle$ via an intermediate state $|\mathrm{i}\rangle$. The two-photon line strength is given by a weighted sum of one-photon transition routes via different intermediate states $|\mathrm{i}\rangle$. Their weights-illustrated here by different grey tones-are determined by the inverse of the energy mismatch $\hbar\left(\omega_{\mathrm{ig}}-\omega_{1}\right)$ between the photon energy $\hbar \omega_{1}$ and the transition energy $\hbar \omega_{\text {ig. }}$. (b) Two-photon transitions between two $\Sigma$-states may occur via $\Sigma$ or $\Pi$ intermediate states. Transition routes are labeled by the effective electric-dipole matrix element $\left(\mu_{\|} \boldsymbol{\mu}_{\|}^{\prime}, \mu_{+} \boldsymbol{\mu}_{-}^{\prime}, \mu_{-}^{\prime \prime} \mu_{+}^{\prime \prime}\right.$, see Eq. (25)) and the relevant spherical tensor component of the electric-dipole operator in the molecule-fixed frame $\left(\tau_{1,2}=0, \pm 1\right)$.

principle on both the vibronic and the nuclear-spin-rotational states of the intermediate level. However, since the nuclearspin-rotational contribution to $\omega_{\mathrm{i}_{\mathrm{ev}} \mathrm{i}_{\text {nsr }} \mathrm{g}}$ is small compared to the vibronic one, we may neglect the latter and approximate the frequency mismatch for far-off-resonant excitation $^{20}$ as $\omega_{\mathrm{iev}_{\mathrm{insrg}}}-\omega_{1} \approx \omega_{\mathrm{ievg}}-\omega_{1}$. Doing so, the term $1 /\left(\omega_{\mathrm{i}_{\mathrm{ev}} \mathrm{i}_{\mathrm{nsrg}}}-\omega_{1}\right) \approx 1 /\left(\omega_{\mathrm{i}_{\mathrm{evg}}}-\omega_{1}\right)$ may be factored out of the sum over the nuclear-spin-rotational intermediate states $\left(\sum_{\mathrm{i}_{\mathrm{nsr}}}\right)$ and the expression for the line strength separates into a product of two independent sums, $\sum_{\mathrm{i}_{\mathrm{ev}}}$ and $\sum_{\mathrm{i}_{\mathrm{nsr}}}$,

$$
\begin{aligned}
S_{\mathrm{ge}}= & \sum_{M_{\mathrm{e}}, M_{\mathrm{g}}} \mid \sum_{\tau_{1}, \tau_{2}}\left(\sum_{\mathrm{i}_{\mathrm{ev}}} \frac{1}{\omega_{\mathrm{ievg}_{\mathrm{ev}}}-\omega_{1}}\left\langle\mathrm{e}_{\mathrm{ev}}\left|\mathrm{T}_{\tau_{1}}^{1}[\boldsymbol{\mu}]\right| \mathrm{i}_{\mathrm{ev}}\right\rangle\right. \\
& \left.\times\left\langle\mathrm{i}_{\mathrm{ev}}\left|\mathrm{T}_{\tau_{2}}^{1}[\boldsymbol{\mu}]\right| \mathrm{g}_{\mathrm{ev}}\right\rangle\right)\left(\sum_{\mathrm{i}_{\mathrm{nsr}}}\left\langle\mathrm{e}_{\mathrm{nsr}}\left|\left[\mathcal{D}_{\sigma \tau_{1}}^{(1)}\right]^{*}\right| \mathrm{i}_{\mathrm{nsr}}\right\rangle\right. \\
& \left.\times\left\langle\mathrm{i}_{\mathrm{nsr}}\left|\left[\mathcal{D}_{\sigma \tau_{2}}^{(1)}\right]^{*}\right| \mathrm{g}_{\mathrm{nsr}}\right\rangle\right)\left.\right|^{2} .
\end{aligned}
$$

Without the energy-mismatch weighting factors, the sum over the intermediate nuclear-spin-rotational states is a sum of projection operators $\left|i_{\text {nsr }}\right\rangle\left\langle i_{\text {nsr }}\right|$. Since this sum includes all nuclear-spin-rotational states, it is equal to the identity operator $I_{\mathrm{nsr}}$ for the nuclear-spin-rotational states, i.e.,

$$
\sum_{\mathrm{i}_{\text {nsr }}}\left|\mathrm{i}_{\mathrm{nsr}}\right\rangle\left\langle\mathrm{i}_{\mathrm{nsr}}\right|=I_{\mathrm{nsr}} \text {. }
$$

Therefore, we arrive at

$$
\begin{aligned}
S_{\mathrm{ge}}= & \sum_{M_{\mathrm{e}}, M_{\mathrm{g}}} \mid \sum_{\tau_{1}, \tau_{2}}\left\langle\mathrm{e}_{\mathrm{nsr}}\left|\left[\mathcal{D}_{\sigma \tau_{1}}^{(1)}\right]^{*}\left[\mathcal{D}_{\sigma \tau_{2}}^{(1)}\right]^{*}\right| \mathrm{g}_{\mathrm{nsr}}\right\rangle \\
& \times \sum_{\mathrm{i}_{\mathrm{ev}}} \frac{1}{\omega_{\mathrm{i}_{\mathrm{evg}} \mathrm{g}}-\omega_{1}}\left\langle\mathrm{e}_{\mathrm{ev}}\left|\mathrm{T}_{\tau_{1}}^{1}[\boldsymbol{\mu}]\right| \mathrm{i}_{\mathrm{ev}}\right\rangle \\
& \times\left.\left\langle\mathrm{i}_{\mathrm{ev}}\left|\mathrm{T}_{\tau_{2}}^{1}[\boldsymbol{\mu}]\right| \mathrm{g}_{\mathrm{ev}}\right\rangle\right|^{2} .
\end{aligned}
$$

To proceed, we need to choose a basis for the molecular states. We focus on the frequent case of transitions between ${ }^{1} \Sigma$ states (and note that the following treatment can be adapted to other state symmetries and coupling cases, see Refs. 16 and 17). We chose the Hund's case (b) notation for the electronic-vibrational ground, intermediate, and excited states,

$$
\begin{aligned}
& \left|\mathrm{g}_{\mathrm{ev}}\right\rangle=\left|n^{\prime \prime} \Lambda^{\prime \prime}, v^{\prime \prime}\right\rangle, \\
& \left|\mathrm{i}_{\mathrm{ev}}\right\rangle=\left|n_{\mathrm{i}_{\mathrm{ev}}} \Lambda_{\mathrm{i}_{\mathrm{ev}}}, v_{\mathrm{ev}}\right\rangle, \\
& \left|\mathrm{e}_{\mathrm{ev}}\right\rangle=\left|n^{\prime} \Lambda^{\prime}, v^{\prime}\right\rangle .
\end{aligned}
$$

Here, $n^{\prime \prime}, n_{\mathrm{iev}}$, and $n^{\prime}$ denote the electronic ground, intermediate, and excited levels, respectively. $v^{\prime \prime}, v_{\mathrm{iev}}, v^{\prime}$ stand for quantum numbers of the corresponding vibrational states and $\Lambda^{\prime \prime}, \Lambda_{\mathrm{iev}}, \Lambda^{\prime}$ are the projection quantum numbers of the total electron orbital angular momenta on the internuclear axis. Refer to Table I for a summary of the symbols used in the present work.

The angular part of the ground and excited states are written as

$$
\begin{aligned}
& \left|\mathrm{g}_{\mathrm{nsr}}\right\rangle=\left|N^{\prime \prime} \Lambda^{\prime \prime} I^{\prime \prime} F^{\prime \prime} M_{F}^{\prime \prime}\right\rangle, \\
& \left|\mathrm{e}_{\mathrm{nsr}}\right\rangle=\left|N^{\prime} \Lambda^{\prime} I^{\prime} F^{\prime} M_{F}^{\prime}\right\rangle,
\end{aligned}
$$

with $N^{\prime \prime}$ and $N^{\prime}$ the rotational quantum numbers in the ground and the excited states, $I^{\prime \prime}$ and $I^{\prime}$ the respective nuclear spin quantum numbers, $F^{\prime \prime}$ and $F^{\prime}$ the total angular momentum quantum numbers as well as $M_{F}^{\prime \prime}, M_{F}^{\prime}$ the corresponding angular momentum projection quantum numbers.

As we are assuming $\Sigma$ states for the ground and the excited state, we have $\Lambda^{\prime \prime}=\Lambda^{\prime}=0$. For the intermediate state, also states with $\Lambda_{\mathrm{i}_{\mathrm{ev}}} \neq 0$ need to be considered (see Fig. 2(b)).

Using this notation, the line strength for the two-photon transition is

$$
\begin{aligned}
S_{\prime \prime \leftrightarrow^{\prime}}= & \sum_{M_{F}^{\prime}, M_{F}^{\prime \prime}} \mid \sum_{\tau_{1}, \tau_{2}}\left\langle N^{\prime} \Lambda^{\prime} I^{\prime} F^{\prime} M_{F}^{\prime}\left|\left[\mathcal{D}_{\sigma \tau_{1}}^{(1)}\right]^{*}\left[\mathcal{D}_{\sigma \tau_{2}}^{(1)}\right]^{*}\right| N^{\prime \prime} \Lambda^{\prime \prime} I^{\prime \prime} F^{\prime \prime} M_{F}^{\prime \prime}\right\rangle \\
& \times\left.\sum_{\mathrm{i}_{\mathrm{ev}}} \frac{1}{\omega_{\mathrm{iev}}-\omega_{1}}\left\langle n^{\prime} \Lambda^{\prime}, v^{\prime}\left|\mathrm{T}_{\tau_{1}}^{1}[\boldsymbol{\mu}]\right| n_{\mathrm{i}_{\mathrm{ev}}} \Lambda_{\mathrm{i}_{\mathrm{ev}}}, v_{\mathrm{i}_{\mathrm{ev}}}\right\rangle\left\langle n_{\mathrm{i}_{\mathrm{ev}}} \Lambda_{\mathrm{i}_{\mathrm{ev}}}, v_{\mathrm{i}_{\mathrm{ev}}}\left|\mathrm{T}_{\tau_{2}}^{1}[\boldsymbol{\mu}]\right| n^{\prime \prime} \Lambda^{\prime \prime}, v^{\prime \prime}\right\rangle\right|^{2} .
\end{aligned}
$$

Exploiting that the nuclear-spin states are not affected in electric-dipole transitions, we decouple the nuclear spin from the total angular momentum in the ground state according to 
TABLE I. Symbols used in the model of the $\left[2+1^{\prime}\right]$ REMPI process.

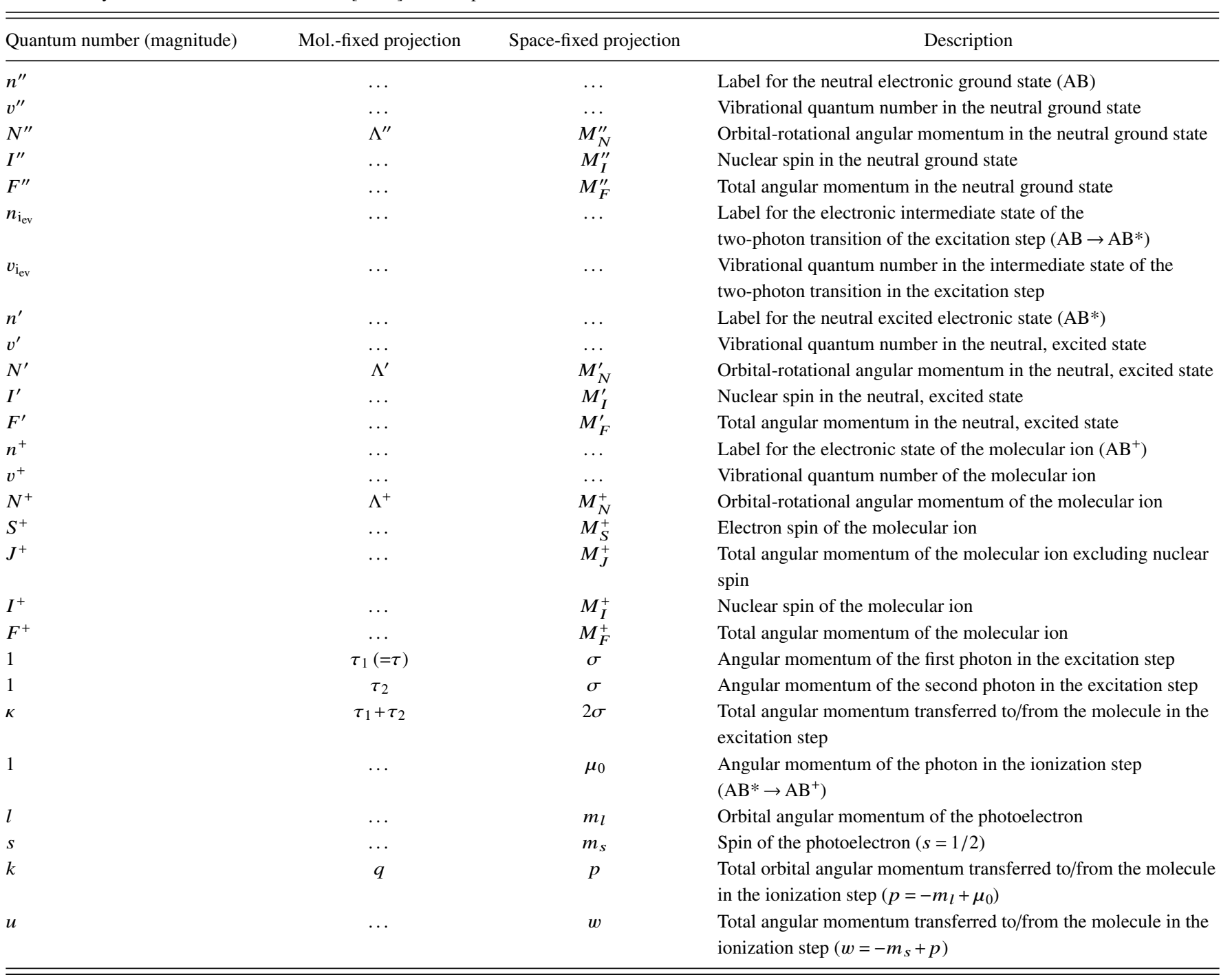

$$
\begin{aligned}
& \left|N^{\prime \prime} \Lambda^{\prime \prime} I^{\prime \prime} F^{\prime \prime} M_{F}^{\prime \prime}\right\rangle \\
& \quad=\sum_{M_{N}^{\prime \prime}, M_{I}^{\prime \prime}} C_{N^{\prime \prime} M_{N}^{\prime \prime} I^{\prime \prime} M_{I}^{\prime \prime}}^{\left.F^{\prime \prime} N^{\prime \prime} \Lambda^{\prime \prime} M_{N}^{\prime \prime}, I^{\prime \prime} M_{I}^{\prime \prime}\right\rangle}
\end{aligned}
$$

with the Clebsch-Gordan coefficients $C_{N^{\prime \prime} M_{N}^{\prime \prime} I^{\prime \prime} M_{I}^{\prime \prime}}^{F^{\prime \prime}}$ and analogously in the excited state.

The angular matrix element in Eq. (12) thus accounts for

$$
\begin{aligned}
& \left\langle N^{\prime} \Lambda^{\prime} I^{\prime} F^{\prime} M_{F}^{\prime}\left|\left[\mathcal{D}_{\sigma \tau_{1}}^{(1)}\right]^{*}\left[\mathcal{D}_{\sigma \tau_{2}}^{(1)}\right]^{*}\right| N^{\prime \prime} \Lambda^{\prime \prime} I^{\prime \prime} F^{\prime \prime} M_{F}^{\prime \prime}\right\rangle
\end{aligned}
$$

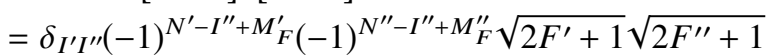

$$
\begin{aligned}
& \times \sum_{M_{N}^{\prime}, M_{N}^{\prime \prime}}\left\langle N^{\prime} \Lambda^{\prime} M_{N}^{\prime}\left|\left[\mathcal{D}_{\sigma \tau_{1}}^{(1)}\right]^{*}\left[\mathcal{D}_{\sigma \tau_{2}}^{(1)}\right]^{*}\right| N^{\prime \prime} \Lambda^{\prime \prime} M_{N}^{\prime \prime}\right\rangle \\
& \times \sum_{M_{I}^{\prime \prime}}\left(\begin{array}{ccc}
N^{\prime} & I^{\prime \prime} & F^{\prime} \\
M_{N}^{\prime} & M_{I}^{\prime \prime} & -M_{F}^{\prime}
\end{array}\right)\left(\begin{array}{ccc}
N^{\prime \prime} & I^{\prime \prime} & F^{\prime \prime} \\
M_{N}^{\prime \prime} & M_{I}^{\prime \prime} & -M_{F}^{\prime \prime}
\end{array}\right),
\end{aligned}
$$

where the orthonormality of the nuclear spin states has been used and the Clebsch-Gordan coefficients have been replaced by $3 \mathrm{j}$-symbols.
The rotational matrix element on the next-to-last line in Eq. (14) can be reformulated using the relation ${ }^{21,22}$

$$
\begin{aligned}
\mathcal{D}_{m_{1}^{\prime} m_{1}}^{\left(j_{1}\right)} \mathcal{D}_{m_{2}^{\prime} m_{2}}^{\left(j_{2}\right)}= & \sum_{j_{3}=\left|j_{1}-j_{2}\right|}^{j_{1}+j_{2}}\left(2 j_{3}+1\right)\left(\begin{array}{ccc}
j_{1} & j_{2} & j_{3} \\
m_{1}^{\prime} & m_{2}^{\prime} & m_{3}^{\prime}
\end{array}\right) \\
& \times\left(\begin{array}{ccc}
j_{1} & j_{2} & j_{3} \\
m_{1} & m_{2} & m_{3}
\end{array}\right)\left[\mathcal{D}_{m_{3}^{\prime} m_{3}}^{\left(j_{3}\right)}\right]^{*},
\end{aligned}
$$

as $^{17,23}$

$$
\begin{aligned}
\left\langle N^{\prime} \Lambda^{\prime} M_{N}^{\prime}\left|\left[\mathcal{D}_{\sigma \tau_{1}}^{(1)}\right]^{*}\left[\mathcal{D}_{\sigma \tau_{2}}^{(1)}\right]^{*}\right| N^{\prime \prime} \Lambda^{\prime \prime} M_{N}^{\prime \prime}\right\rangle \\
=\sum_{\kappa=0}^{2}(2 \kappa+1)\left(\begin{array}{ccc}
1 & 1 & \kappa \\
-\sigma & -\sigma & 2 \sigma
\end{array}\right)\left(\begin{array}{ccc}
1 & 1 & \kappa \\
-\tau_{2} & -\tau_{1} & \tau_{1}+\tau_{2}
\end{array}\right) \\
\quad \times\left\langle N^{\prime} \Lambda^{\prime} M_{N}^{\prime}\left|\mathcal{D}_{-2 \sigma,-\tau_{1}-\tau_{2}}^{(\kappa)}\right| N^{\prime \prime} \Lambda^{\prime \prime} M_{N}^{\prime \prime}\right\rangle
\end{aligned}
$$

Inserting appropriately normalized Wigner rotation matrices for the rotational states (with the three Euler angles $\phi, \theta, \chi)$,

$$
\left\langle\phi \theta \chi \mid N \Lambda M_{N}\right\rangle=\sqrt{\frac{2 N+1}{8 \pi^{2}}}\left[\mathcal{D}_{M_{N} \Lambda}^{(N)}(\phi, \theta, \chi)\right]^{*},
$$


we obtain for the matrix element in Eq. (16) an integral over three Wigner rotation matrices that may be expressed in the form of $3 \mathrm{j}$-symbols as ${ }^{21,22}$

$$
\begin{aligned}
\left\langle N^{\prime} \Lambda^{\prime}\right. & \left.M_{N}^{\prime}\left|\mathcal{D}_{-2 \sigma,-\tau_{1}-\tau_{2}}^{(\kappa)}\right| N^{\prime \prime} \Lambda^{\prime \prime} M_{N}^{\prime \prime}\right\rangle \\
= & \sqrt{2 N^{\prime}+1} \sqrt{2 N^{\prime \prime}+1}(-1)^{M_{N}^{\prime \prime}-\Lambda^{\prime \prime}} \\
& \times\left(\begin{array}{ccc}
N^{\prime} & \kappa & N^{\prime \prime} \\
M_{N}^{\prime} & -2 \sigma & -M_{N}^{\prime \prime}
\end{array}\right)\left(\begin{array}{ccc}
N^{\prime} & \kappa & N^{\prime \prime} \\
\Lambda^{\prime} & -\tau_{1}-\tau_{2} & -\Lambda^{\prime \prime}
\end{array}\right) .
\end{aligned}
$$

Substituting this expression into Eq. (16) yields

$$
\begin{aligned}
\left\langle N^{\prime} \Lambda^{\prime} M_{N}^{\prime}\left|\left[\mathcal{D}_{\sigma \tau_{1}}^{(1)}\right]^{*}\left[\mathcal{D}_{\sigma \tau_{2}}^{(1)}\right]^{*}\right| N^{\prime \prime} \Lambda^{\prime \prime} M_{N}^{\prime \prime}\right\rangle & \\
= & \sqrt{2 N^{\prime}+1} \sqrt{2 N^{\prime \prime}+1}(-1)^{M_{N}^{\prime \prime}-\Lambda^{\prime \prime}} \\
& \times \sum_{\kappa=0}^{2}(2 \kappa+1)\left(\begin{array}{ccc}
1 & 1 & \kappa \\
-\sigma & -\sigma & 2 \sigma
\end{array}\right)\left(\begin{array}{ccc}
1 & 1 & \kappa \\
-\tau_{2} & -\tau_{1} & \tau_{1}+\tau_{2}
\end{array}\right) \\
& \times\left(\begin{array}{cccc}
N^{\prime} & \kappa & N^{\prime \prime} \\
M_{N}^{\prime} & -2 \sigma & -M_{N}^{\prime \prime}
\end{array}\right)\left(\begin{array}{ccc}
N^{\prime} & \kappa & N^{\prime \prime} \\
\Lambda^{\prime} & -\tau_{1}-\tau_{2} & -\Lambda^{\prime \prime}
\end{array}\right), \quad(19)
\end{aligned}
$$

and subsequent substitution into Eq. (14) gives

$$
\begin{aligned}
& \left\langle N^{\prime} \Lambda^{\prime} I^{\prime} F^{\prime} M_{F}^{\prime}\left|\left[\mathcal{D}_{\sigma \tau_{1}}^{(1)}\right]^{*}\left[\mathcal{D}_{\sigma \tau_{2}}^{(1)}\right]^{*}\right| N^{\prime \prime} \Lambda^{\prime \prime} I^{\prime \prime} F^{\prime \prime} M_{F}^{\prime \prime}\right\rangle
\end{aligned}
$$

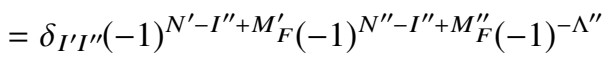

$$
\begin{aligned}
& \times \sqrt{2 F^{\prime}+1} \sqrt{2 F^{\prime \prime}+1} \sqrt{2 N^{\prime}+1} \sqrt{2 N^{\prime \prime}+1} \\
& \times \sum_{\kappa=0}^{2}(2 \kappa+1)\left(\begin{array}{ccc}
1 & 1 & \kappa \\
-\sigma & -\sigma & 2 \sigma
\end{array}\right)\left(\begin{array}{ccc}
1 & 1 & \kappa \\
-\tau_{2} & -\tau_{1} & \tau_{1}+\tau_{2}
\end{array}\right) \\
& \times\left(\begin{array}{ccc}
N^{\prime} & \kappa & N^{\prime \prime} \\
\Lambda^{\prime} & -\tau_{1}-\tau_{2} & -\Lambda^{\prime \prime}
\end{array}\right)_{M_{N}^{\prime}, M_{N}^{\prime \prime}, M_{I}^{\prime \prime}}(-1)^{M_{N}^{\prime \prime}} \\
& \times\left(\begin{array}{ccc}
N^{\prime} & \kappa & N^{\prime \prime} \\
M_{N}^{\prime} & -2 \sigma & -M_{N}^{\prime \prime}
\end{array}\right)\left(\begin{array}{ccc}
N^{\prime} & I^{\prime \prime} & F^{\prime} \\
M_{N}^{\prime} & M_{I}^{\prime \prime} & -M_{F}^{\prime}
\end{array}\right) \\
& \times\left(\begin{array}{ccc}
N^{\prime \prime} & I^{\prime \prime} & F^{\prime \prime} \\
M_{N}^{\prime \prime} & M_{I}^{\prime \prime} & -M_{F}^{\prime \prime}
\end{array}\right)
\end{aligned}
$$

The sum over the last three 3j-symbols in Eq. (20) may be expressed in terms of a Wigner $6 \mathrm{j}$-symbol ${ }^{21}$ yielding

$$
\begin{aligned}
&\left\langle N^{\prime} \Lambda^{\prime} I^{\prime} F^{\prime} M_{F}^{\prime}\left|\left[\mathcal{D}_{\sigma \tau_{1}}^{(1)}\right]^{*}\left[\mathcal{D}_{\sigma \tau_{2}}^{(1)}\right]^{*}\right| N^{\prime \prime} \Lambda^{\prime \prime} I^{\prime \prime} F^{\prime \prime} M_{F}^{\prime \prime}\right\rangle \\
&= \delta_{I^{\prime} I^{\prime \prime}}(-1)^{N^{\prime}+N^{\prime \prime}-\Lambda^{\prime \prime}-3 I^{\prime \prime}-F^{\prime}-F^{\prime \prime}+M_{F}^{\prime}+2 M_{F}^{\prime \prime}} \\
& \times \sqrt{2 F^{\prime}+1} \sqrt{2 F^{\prime \prime}+1} \sqrt{2 N^{\prime}+1} \sqrt{2 N^{\prime \prime}+1} \\
& \times \sum_{\kappa=0}^{2}(2 \kappa+1)\left(\begin{array}{ccc}
1 & 1 & \kappa \\
-\sigma & -\sigma & 2 \sigma
\end{array}\right)\left(\begin{array}{ccc}
1 & 1 & \kappa \\
-\tau_{2} & -\tau_{1} & \tau_{1}+\tau_{2}
\end{array}\right) \\
& \times\left(\begin{array}{ccc}
N^{\prime} & \kappa & N^{\prime \prime} \\
\Lambda^{\prime} & -\tau_{1}-\tau_{2} & -\Lambda^{\prime \prime}
\end{array}\right)\left\{\begin{array}{ccc}
\kappa & N^{\prime \prime} & N^{\prime} \\
I^{\prime \prime} & F^{\prime} & F^{\prime \prime}
\end{array}\right\} \\
& \times\left(\begin{array}{ccc}
F^{\prime} & \kappa & F^{\prime \prime} \\
-M_{F}^{\prime} & 2 \sigma & M_{F}^{\prime \prime}
\end{array}\right) .
\end{aligned}
$$

Because of the third $3 \mathrm{j}$-symbol in Eq. (21), this matrix element vanishes unless the condition $\tau_{1}+\tau_{2}=\Lambda^{\prime}-\Lambda^{\prime \prime}(=0$ for $\Sigma-\Sigma$ transitions) is met. Hence, when substituting this matrix element into Eq. (12), only terms fulfilling this relation contribute to the sums over $\tau_{1}$ and $\tau_{2}$. We thus skip the sum over $\tau_{2}$ in Eq. (12) by means of the substitution $\tau_{2}=-\tau_{1}$ and drop the index on $\tau_{1}$ by setting $\tau:=\tau_{1}$. Furthermore, the cross terms in the sum over $\kappa$ in Eq. (12) vanish when summing over $M_{F}^{\prime}$ and $M_{F}^{\prime \prime}$ owing to the orthogonality properties of the last $3 \mathrm{j}$-symbol in Eq. (21). As a result we obtain the two-photon line strength as

$$
\begin{aligned}
S_{\prime \prime \leftrightarrow}= & \left(2 F^{\prime}+1\right)\left(2 F^{\prime \prime}+1\right)\left(2 N^{\prime}+1\right)\left(2 N^{\prime \prime}+1\right) \delta_{I^{\prime} I^{\prime \prime}} \\
& \times \sum_{\kappa=0,2}(2 \kappa+1)\left(\begin{array}{ccc}
1 & 1 & \kappa \\
-\sigma & -\sigma & 2 \sigma
\end{array}\right)^{2} \\
& \times\left\{\begin{array}{ccc}
\kappa & N^{\prime \prime} & N^{\prime} \\
I^{\prime \prime} & F^{\prime} & F^{\prime \prime}
\end{array}\right\}^{2}\left(\begin{array}{ccc}
N^{\prime} & \kappa & N^{\prime \prime} \\
0 & 0 & 0
\end{array}\right)^{2} U(\kappa),
\end{aligned}
$$

with

$$
\begin{aligned}
& U(\kappa)=\mid \sum_{\tau=-1}^{1}\left(\begin{array}{ccc}
1 & 1 & \kappa \\
\tau & -\tau & 0
\end{array}\right) \sum_{\mathrm{i}_{\mathrm{ev}}} \frac{1}{\omega_{\mathrm{iev}}-\omega_{1}} \\
& \times\left\langle n^{\prime} \Lambda^{\prime}=0, v^{\prime}\left|\mathrm{T}_{\tau}^{1}[\boldsymbol{\mu}]\right| n_{\mathrm{i}_{\mathrm{ev}}} \Lambda_{\mathrm{i}_{\mathrm{ev}}}, v_{\mathrm{i}_{\mathrm{ev}}}\right\rangle \\
& \times\left.\left\langle n_{\mathrm{i}_{\mathrm{ev}}} \Lambda_{\mathrm{i}_{\mathrm{ev}}}, v_{\mathrm{i}_{\mathrm{ev}}}\left|\mathrm{T}_{-\tau}^{1}[\boldsymbol{\mu}]\right| n^{\prime \prime} \Lambda^{\prime \prime}=0, v^{\prime \prime}\right\rangle\right|^{2} .
\end{aligned}
$$

Since the first $3 \mathrm{j}$-symbol in Eq. (22) vanishes for $\kappa=1$, we have omitted the $\kappa=1$-term in this equation. We study the expression $U(\kappa)$ separately for $\kappa=0$ and $\kappa=2$. For $\kappa=0$, evaluation of the $3 \mathrm{j}$-symbol in Eq. (23) yields

$$
U(0)=\frac{1}{3}\left|\mu_{\|} \mu_{\|}^{\prime}-\mu_{+} \mu_{-}^{\prime}-\mu_{-}^{\prime \prime} \mu_{+}^{\prime \prime}\right|^{2}=\frac{1}{3} \mu_{\mathrm{I}}^{2},
$$

with the abbreviations (see Refs. 13 and 24$)^{25}$

$$
\begin{aligned}
& \mu_{\|} \mu_{\|}^{\prime}=\sum_{\mathrm{i}_{\mathrm{ev}}} \frac{1}{\omega_{\mathrm{i}_{\mathrm{ev}} \mathrm{g}}-\omega_{1}}\left\langle n^{\prime} \Lambda^{\prime}, v^{\prime}\left|\mathrm{T}_{0}^{1}[\boldsymbol{\mu}]\right| n_{\mathrm{i}_{\mathrm{ev}}} \Lambda_{\mathrm{i}_{\mathrm{ev}}}, v_{\mathrm{i}_{\mathrm{ev}}}\right\rangle \\
& \times\left\langle n_{\mathrm{iev}} \Lambda_{\mathrm{i}_{\mathrm{ev}}}, v_{\mathrm{iev}_{\mathrm{ev}}}\left|\mathrm{T}_{0}^{1}[\boldsymbol{\mu}]\right| n^{\prime \prime} \Lambda^{\prime \prime}, v^{\prime \prime}\right\rangle, \\
& \mu_{+} \mu_{-}^{\prime}=\sum_{\mathrm{i}_{\mathrm{ev}}} \frac{1}{\omega_{\mathrm{i}_{\mathrm{ev}} \mathrm{g}}-\omega_{1}}\left\langle n^{\prime} \Lambda^{\prime}, v^{\prime}\left|\mathrm{T}_{+1}^{1}[\boldsymbol{\mu}]\right| n_{\mathrm{i}_{\mathrm{ev}}} \Lambda_{\mathrm{i}_{\mathrm{ev}}}, v_{\mathrm{i}_{\mathrm{ev}}}\right\rangle \\
& \times\left\langle n_{\mathrm{i}_{\mathrm{ev}}} \Lambda_{\mathrm{i}_{\mathrm{ev}}}, v_{\mathrm{i}_{\mathrm{ev}}}\left|\mathrm{T}_{-1}^{1}[\boldsymbol{\mu}]\right| n^{\prime \prime} \Lambda^{\prime \prime}, v^{\prime \prime}\right\rangle, \\
& \mu_{-}^{\prime \prime} \mu_{+}^{\prime \prime}=\sum_{\mathrm{i}_{\mathrm{ev}}} \frac{1}{\omega_{\mathrm{i}_{\mathrm{ev}} \mathrm{g}}-\omega_{1}}\left\langle n^{\prime} \Lambda^{\prime}, v^{\prime}\left|\mathrm{T}_{-1}^{1}[\boldsymbol{\mu}]\right| n_{\mathrm{e}_{\mathrm{ev}}} \Lambda_{\mathrm{i}_{\mathrm{ev}}}, v_{\mathrm{i}_{\mathrm{ev}}}\right\rangle \\
& \times\left\langle n_{\mathrm{i}_{\mathrm{ev}}} \Lambda_{\mathrm{i}_{\mathrm{ev}}}, v_{\mathrm{i}_{\mathrm{ev}}}\left|\mathrm{T}_{+1}^{1}[\boldsymbol{\mu}]\right| n^{\prime \prime} \Lambda^{\prime \prime}, v^{\prime \prime}\right\rangle,
\end{aligned}
$$

and

$$
\mu_{\mathrm{I}}^{2}=\left|\mu_{\|} \mu_{\|}^{\prime}-\mu_{+} \mu_{-}^{\prime}-\mu_{-}^{\prime \prime} \mu_{+}^{\prime \prime}\right|^{2}
$$

For $\kappa=2$, we obtain similarly

$$
U(2)=\frac{1}{30}\left|2 \mu_{\|} \mu_{\|}^{\prime}+\mu_{+} \mu_{-}^{\prime}+\mu_{-}^{\prime \prime} \mu_{+}^{\prime \prime}\right|^{2}=\frac{1}{30} \mu_{\mathrm{S}}^{2},
$$

where we have set $\mu_{\mathrm{S}}^{2}=\left|2 \mu_{\|} \mu_{\|}^{\prime}+\mu_{+} \mu_{-}^{\prime}+\mu_{-}^{\prime \prime} \mu_{+}^{\prime \prime}\right|^{2}$.

The hfs-resolved two-photon line strength between ${ }^{1} \Sigma$ states is thus 


$$
\begin{aligned}
& S_{\prime \prime \leftrightarrow}=\left(2 F^{\prime}+1\right)\left(2 F^{\prime \prime}+1\right)\left(2 N^{\prime}+1\right)\left(2 N^{\prime \prime}+1\right) \delta_{I^{\prime} I^{\prime \prime}}\left[\frac{1}{3} \frac{1}{2 N^{\prime \prime}+1}\left(\begin{array}{ccc}
1 & 1 & 0 \\
-\sigma & -\sigma & 2 \sigma
\end{array}\right)^{2}\left\{\begin{array}{ccc}
0 & N^{\prime \prime} & N^{\prime} \\
I^{\prime \prime} & F^{\prime} & F^{\prime \prime}
\end{array}\right\}^{2} \mu_{\mathrm{I}}^{2}\right. \\
& \left.+\frac{1}{6}\left(\begin{array}{ccc}
N^{\prime} & 2 & N^{\prime \prime} \\
0 & 0 & 0
\end{array}\right)^{2}\left(\begin{array}{ccc}
1 & 1 & 2 \\
-\sigma & -\sigma & 2 \sigma
\end{array}\right)^{2}\left\{\begin{array}{ccc}
2 & N^{\prime \prime} & N^{\prime} \\
I^{\prime \prime} & F^{\prime} & F^{\prime \prime}
\end{array}\right\}^{2} \mu_{\mathrm{S}}^{2}\right] \text {. }
\end{aligned}
$$

For circular polarized radiation, we have $\sigma= \pm 1$. As $\sigma$ denotes the projection associated with $\kappa$ on the space-fixed $z$-axis, we must have $\kappa \geq \sigma$. Hence, the term with $\kappa=0$ in Eq. (22), i.e., the first summand within brackets in Eq. (28), does not apply for circular polarization. With the last $3 \mathrm{j}$-symbol in Eq. (28) accounting for $1 / 5$, we thus obtain

$$
S_{\prime \prime \leftrightarrow}^{(\text {circ })}=\frac{1}{30}\left(2 F^{\prime}+1\right)\left(2 F^{\prime \prime}+1\right)\left(2 N^{\prime}+1\right)\left(2 N^{\prime \prime}+1\right) \delta_{I^{\prime} I^{\prime \prime}}\left(\begin{array}{ccc}
N^{\prime} & 2 & N^{\prime \prime} \\
0 & 0 & 0
\end{array}\right)^{2}\left\{\begin{array}{ccc}
2 & N^{\prime \prime} & N^{\prime} \\
I^{\prime \prime} & F^{\prime} & F^{\prime \prime}
\end{array}\right\}^{2} \mu_{\mathrm{S}}^{2} .
$$

For linear polarization, we have $\sigma=0$ for a suitable chosen space-fixed frame of reference. The two squared $3 \mathrm{j}$ symbols involving $\sigma$ in Eq. (28) then account for $1 / 3$ and $2 / 15$, respectively, and the line strength is

$$
\begin{aligned}
S_{\prime^{\prime}}^{(\text {lin })}= & \left(2 F^{\prime}+1\right)\left(2 F^{\prime \prime}+1\right)\left(2 N^{\prime}+1\right)\left(2 N^{\prime \prime}+1\right) \delta_{I^{\prime} I^{\prime \prime}} \\
& \times\left[\frac{1}{9} \frac{1}{2 N^{\prime \prime}+1}\left\{\begin{array}{ccc}
0 & N^{\prime \prime} & N^{\prime} \\
I^{\prime \prime} & F^{\prime} & F^{\prime \prime}
\end{array}\right\}^{2} \mu_{\mathrm{I}}^{2}\right. \\
& \left.+\frac{1}{45}\left(\begin{array}{ccc}
N^{\prime} & 2 & N^{\prime \prime} \\
0 & 0 & 0
\end{array}\right)^{2}\left\{\begin{array}{ccc}
2 & N^{\prime \prime} & N^{\prime} \\
I^{\prime \prime} & F^{\prime} & F^{\prime \prime}
\end{array}\right\}^{2} \mu_{\mathrm{S}}^{2}\right] .
\end{aligned}
$$

Summing the above expression over all hyperfine components corresponding to a specific rotational transition reproduces the results for the rotationally resolved two-photon line strength reported in Ref. 13.

So far, the line strength associated with the total population in a $\left(N^{\prime}, I^{\prime}, F^{\prime}\right)$ level has been considered. This is the quantity usually of interest for transitions between bound states. For our particular purpose, namely, to describe the REMPI process, the population in a certain Zeeman state $\left|N^{\prime}, I^{\prime}, F^{\prime}, M_{F}^{\prime}\right\rangle$ of the neutral, electronically excited state is needed. The relevant quantity is thus $^{26}$

$$
S_{\mathrm{ge}}\left(M_{\mathrm{e}}\right)=\sum_{M_{\mathrm{g}}}\left|\sum_{\mathrm{i}} \frac{1}{\omega_{\mathrm{ig}}-\omega_{1}}\left\langle\mathrm{e}\left|\mathbf{e}_{\sigma} \cdot \boldsymbol{\mu}\right| \mathrm{i}\right\rangle\left\langle\mathrm{i}\left|\mathbf{e}_{\sigma} \cdot \boldsymbol{\mu}\right| \mathrm{g}\right\rangle\right|^{2} .
$$

For hfs-resolved transitions, this quantity is written in our notation as

$$
\begin{aligned}
S\left(F^{\prime \prime}, F^{\prime}, M_{F}^{\prime}\right)= & \sum_{M_{F}^{\prime \prime}} \mid \sum_{\tau_{1}, \tau_{2}}\left\langle N^{\prime} \Lambda^{\prime} I^{\prime} F^{\prime} M_{F}^{\prime}\left|\left[\mathcal{D}_{\sigma \tau_{1}}^{(1)}\right]^{*}\left[\mathcal{D}_{\sigma \tau_{2}}^{(1)}\right]^{*}\right| N^{\prime \prime} \Lambda^{\prime \prime} I^{\prime \prime} F^{\prime \prime} M_{F}^{\prime \prime}\right\rangle \\
& \times\left.\sum_{\mathrm{i}_{\mathrm{ev}}} \frac{1}{\omega_{\mathrm{ievg}}-\omega_{1}}\left\langle n^{\prime} \Lambda^{\prime}, v^{\prime}\left|\mathrm{T}_{\tau_{1}}^{1}[\mu]\right| n_{\mathrm{iev}_{\mathrm{ev}}} \Lambda_{\mathrm{iev}}, v_{\mathrm{iev}}\right\rangle\left\langle n_{\mathrm{iev}_{\mathrm{ev}}} \Lambda_{\mathrm{i}_{\mathrm{ev}}}, v_{\mathrm{i}_{\mathrm{ev}}}\left|\mathrm{T}_{\tau_{2}}^{1}[\mu]\right| n^{\prime \prime} \Lambda^{\prime \prime}, v^{\prime \prime}\right\rangle\right|^{2},
\end{aligned}
$$

where, as before, the nuclear-spin-rotational contribution to the energy mismatch has been neglected.

Substituting the angular transition matrix element from Eq. (21) and applying the above-mentioned restrictions and substitutions for $\tau_{1}, \tau_{2}$ yields (for $\Sigma$ - $\Sigma$-transitions)

$$
\begin{aligned}
S\left(F^{\prime \prime}, F^{\prime}, M_{F}^{\prime}\right)= & \left(2 F^{\prime}+1\right)\left(2 F^{\prime \prime}+1\right)\left(2 N^{\prime}+1\right)\left(2 N^{\prime \prime}+1\right) \delta_{I^{\prime} I^{\prime \prime}} \sum_{M_{F}^{\prime}} \mid \sum_{\kappa=0,2}(2 \kappa+1)\left(\begin{array}{ccc}
N^{\prime} & \kappa & N^{\prime \prime} \\
0 & 0 & 0
\end{array}\right)\left(\begin{array}{ccc}
1 & 1 & \kappa \\
-\sigma & -\sigma & 2 \sigma
\end{array}\right) \\
& \times\left\{\begin{array}{ccc}
\kappa & N^{\prime \prime} & N^{\prime} \\
I^{\prime \prime} & F^{\prime} & F^{\prime \prime}
\end{array}\right\}\left(\begin{array}{ccc}
F^{\prime} & \kappa & F^{\prime \prime} \\
-M_{F}^{\prime} & 2 \sigma & M_{F}^{\prime \prime}
\end{array}\right) \sum_{\tau}\left(\begin{array}{ccc}
1 & 1 & \kappa \\
\tau & -\tau & 0
\end{array}\right) \sum_{\mathrm{i}_{\mathrm{ev}}} \frac{1}{\omega_{\mathrm{ievg}}-\omega_{1}} \times\left\langle n^{\prime} \Lambda^{\prime}=0, v^{\prime}\left|\mathrm{T}_{\tau}^{1}[\mu]\right| n_{\mathrm{iev}} \Lambda_{\mathrm{i}_{\mathrm{ev}}}, v_{\mathrm{iev}}\right\rangle \\
& \times\left.\left\langle n_{\mathrm{iev}} \Lambda_{\mathrm{iev}}, v_{\mathrm{iev}}\left|\mathrm{T}_{-\tau}^{1}[\mu]\right| n^{\prime \prime} \Lambda^{\prime \prime}=0, v^{\prime \prime}\right\rangle\right|^{2}
\end{aligned}
$$

In contrast to the total line strength considered before, the above expression does not include a sum over the excitedstate projection angular momentum quantum number $M_{F}^{\prime}$. As a consequence, the orthogonality of the $3 \mathrm{j}$-symbols may not be used to eliminate the cross terms, as was possible in the derivation of Eq. (22). Hence, angular terms may not be separated from vibronic ones. Therefore, calculation of relative nuclear-spin-rotational intensities is in general only possible when knowing both the magnitude and the phase of the vibronic transition matrix elements. If the phases are unknown, relative intensities may in general not be determined (see also Sec. IV).

For transitions involving a change in the rotational angular momentum, i.e., $\mathrm{O}$ and $\mathrm{S}$ lines $(\Delta N=-2$ and +2 , 
respectively), however, only the $\kappa=2$ term in Eq. (33) is relevant. We may thus simplify the above result further arriving at

$$
\begin{aligned}
S_{\mathrm{S}, \mathrm{O}}\left(F^{\prime \prime}, F^{\prime}, M_{F}^{\prime}\right) & \\
= & \frac{5}{6}\left(2 F^{\prime}+1\right)\left(2 F^{\prime \prime}+1\right)\left(2 N^{\prime}+1\right)\left(2 N^{\prime \prime}+1\right) \\
& \times \delta_{I^{\prime} I^{\prime \prime}}\left(\begin{array}{ccc}
1 & 1 & 2 \\
-\sigma & -\sigma & 2 \sigma
\end{array}\right)^{2}\left\{\begin{array}{ccc}
2 & N^{\prime \prime} & N^{\prime} \\
I^{\prime \prime} & F^{\prime} & F^{\prime \prime}
\end{array}\right)^{2} \\
& \times \sum_{M_{F}^{\prime \prime}}\left(\begin{array}{ccc}
F^{\prime} & 2 & F^{\prime \prime} \\
-M_{F}^{\prime} & 2 \sigma & M_{F}^{\prime \prime}
\end{array}\right)^{2}\left(\begin{array}{ccc}
N^{\prime} & 2 & N^{\prime \prime} \\
0 & 0 & 0
\end{array}\right)^{2} \mu_{\mathrm{S}}^{2},
\end{aligned}
$$

with $\mu_{\mathrm{S}}^{2}$ as in Eq. (27).

In the case of linear polarized radiation $(\sigma=0)$, this results in

$$
\begin{aligned}
& S_{\mathrm{S}, \mathrm{O}}^{(\operatorname{lin})}\left(F^{\prime \prime}, F^{\prime}, M_{F}^{\prime}\right) \\
&= \frac{1}{9}\left(2 F^{\prime}+1\right)\left(2 F^{\prime \prime}+1\right)\left(2 N^{\prime}+1\right)\left(2 N^{\prime \prime}+1\right) \\
& \times \delta_{I^{\prime} I^{\prime \prime}}\left\{\begin{array}{ccc}
2 & N^{\prime \prime} & N^{\prime} \\
I^{\prime \prime} & F^{\prime} & F^{\prime \prime}
\end{array}\right\}\left(\begin{array}{ccc}
F^{\prime} & 2 & F^{\prime \prime} \\
-M_{F}^{\prime} & 0 & M_{F}^{\prime}
\end{array}\right)^{2} \\
& \times\left(\begin{array}{ccc}
N^{\prime} & 2 & N^{\prime \prime} \\
0 & 0 & 0
\end{array}\right)^{2} \mu_{\mathrm{S}}^{2} .
\end{aligned}
$$

\section{IONIZATION STEP}

\section{A. Effects of anisotropic populations}

Having discussed the excitation step $(\mathrm{AB} \rightarrow \mathrm{AB} *)$, we now turn to the ionization step $\left(\mathrm{AB}^{*} \rightarrow \mathrm{AB}^{+}\right)$.

The population of the neutral, excited molecules $\mathrm{AB}^{*}$ produced in the excitation step is in general anisotropic, i.e., different Zeeman states are unequally populated. This effect is illustrated in Fig. 3 with hyperfine structure omitted for clarity: A diatomic molecule $\mathrm{AB}$ in the neutral ground state is excited by absorption of electromagnetic radiation at an angular frequency $\omega_{1}$ yielding a population $\rho^{\prime}$ of excited molecules $\mathrm{AB}^{*}$. These excited molecules $\mathrm{AB}^{*}$ are then ionized by electromagnetic radiation at another frequency $\omega_{2}$ forming the molecular ions $\mathrm{AB}^{+}$. In the case of excitation with linear $z$-polarized radiation, only transitions without change in the

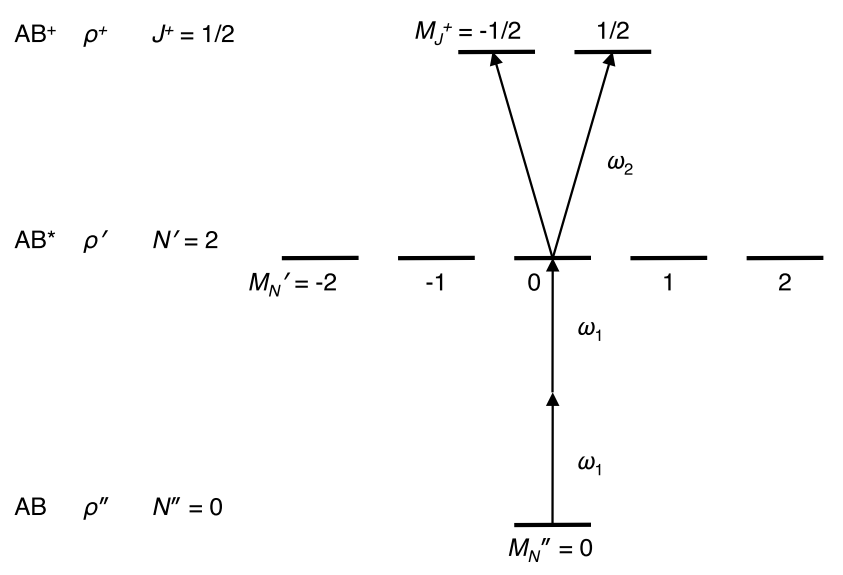

FIG. 3. Anisotropic population in the excited neutral state generated in REMPI: example of the $N^{\prime \prime}=0 \rightarrow N^{\prime}=2 \rightarrow N^{+}=0$ REMPI scheme with linear polarization for excitation. Because of the selection rule $M_{N}^{\prime}=M_{N}^{\prime \prime}$, only the $M_{N}^{\prime}=0$ Zeeman state of the neutral excited level is populated. Ionization may thus only occur from this particular Zeeman state.

projection angular momentum quantum number, i.e., with $M_{N}^{\prime}=M_{N}^{\prime \prime}$, are allowed. Therefore, the entire excited state population $\rho^{\prime}$ is confined to the $M_{N}^{\prime}=0$ Zeeman state. As a consequence, ionization may only occur from this particular Zeeman state and transitions to $\mathrm{AB}^{+}$from other $\mathrm{AB}^{*}$ Zeeman states do not contribute to the ionization process.

In the following, we develop a model for ionization of anisotropically populated levels based on weighting of Zeeman states by their population. First, we only consider spin-rotational fine structure, thereafter we extend our model to cover hyperfine structure as well.

\section{B. Fine structure}

Denoting the population of excited molecules $\mathrm{AB}^{*}$ in a certain Zeeman state by $\rho^{\prime}\left(J^{\prime}, M_{J}^{\prime}\right)$, the quantity $P_{\rho^{\prime}}\left(J^{\prime}, J^{+}\right)$, proportional to the photoionization transition probability between fine-structure levels, is given by

$$
\begin{aligned}
P_{\rho^{\prime}}\left(J^{\prime}, J^{+}\right)= & \sum_{l} \sum_{m_{l}} \sum_{m_{s}} \sum_{M_{J}^{\prime}, M_{J}^{+}} \rho^{\prime}\left(J^{\prime}, M_{J}^{\prime}\right) \\
& \times \mid\left(\left\langle n^{+} \Lambda^{+}, v^{+}, N^{+} \Lambda^{+} S^{+} J^{+} M_{J}^{+}\right|\right. \\
& \times\left.\left\langle s, m_{s}\left|\left\langle l, m_{l}\right|\right) \mu \mid n^{\prime} \Lambda^{\prime}, v^{\prime}, N^{\prime} \Lambda^{\prime} S^{\prime} J^{\prime} M_{J}^{\prime}\right\rangle\right|^{2} .
\end{aligned}
$$

Substituting the matrix element from Eq. (18) in Paper $\mathrm{I},{ }^{10}$ we obtain

$$
\begin{aligned}
P_{\rho^{\prime}}\left(J^{\prime}, J^{+}\right)= & \left(2 N^{\prime}+1\right)\left(2 N^{+}+1\right)\left(2 S^{\prime}+1\right)\left(2 J^{\prime}+1\right)\left(2 J^{+}+1\right) \sum_{l} \sum_{m_{l}} \sum_{m_{s}} \sum_{M_{J}^{\prime}, M_{J}^{+}} \rho^{\prime}\left(J^{\prime}, M_{J}^{\prime}\right) \\
& \times \mid \sum_{k=l \pm 1}(-1)^{k} \sqrt{2 k+1}\left(\begin{array}{ccc}
l & 1 & k \\
-m_{l} & \mu_{0} & -p
\end{array}\right)\left\langle n^{+} \Lambda^{+}, v^{+}\left|\mathrm{T}_{\Delta \Lambda}^{\prime k}\right| n^{\prime} \Lambda^{\prime}, v^{\prime}\right\rangle\left(\begin{array}{ccc}
N^{+} & k & N^{\prime} \\
-\Lambda^{+} & \Delta \Lambda & \Lambda^{\prime}
\end{array}\right) \\
& \times\left.\sum_{u=|k-s|}^{k+s}(2 u+1)\left(\begin{array}{ccc}
J^{+} & u & J^{\prime} \\
-M_{J}^{+} & w & M_{J}
\end{array}\right)\left(\begin{array}{ccc}
u & k & s \\
w & -p & m_{S}
\end{array}\right)\left\{\begin{array}{ccc}
J^{+} & u & J^{\prime} \\
N^{+} & k & N^{\prime} \\
S^{+} & s & S^{\prime}
\end{array}\right)\right|^{2}
\end{aligned}
$$


As the terms in the sum over $M_{J}^{\prime}$ are weighted by the populations $\rho^{\prime}\left(J^{\prime}, M_{J}^{\prime}\right)$, the orthogonality properties of the Wigner $3 \mathrm{j}$-symbols may not be used to eliminate the cross terms in the above expression as is possible for direct ionization (see Paper $\mathrm{I}^{10}$ ). Hence, the vibronic transition matrix elements $\left\langle n^{+} \Lambda^{+}, v^{+}\left|\mathrm{T}_{\Delta \Lambda}^{\prime}\right| n \Lambda, v\right\rangle$ may not be isolated from the other terms in Eq. (37) and, since these matrix elements are in general complex quantities, the transition probability may not be calculated unless the magnitude and the (relative) phases of these matrix elements are known. In other words, for ionization of an anisotropically populated level, interference effects between different vibronic transition matrix elements become important. ${ }^{11,12}$

In order to illustrate this effect, we study the transition probability for a ${ }^{1} \Sigma \rightarrow{ }^{2} \Sigma$ ionization process for the spinrotation levels $N^{\prime}=J^{\prime}=2 \rightarrow N^{+}=2, J^{+}=3 / 2$ and $N^{\prime}$ $=J^{\prime}=2 \rightarrow N^{+}=2, J^{+}=5 / 2$. We assume the population of the neutral $J^{\prime}=2$ level to be confined to the $M_{J}^{\prime}=0$ Zeeman state, i.e., $\rho^{\prime}\left(J^{\prime}=2, M_{J}^{\prime}=0\right)=1$ and $\rho^{\prime}\left(J^{\prime}=2, M_{J}^{\prime} \neq 0\right)=0$, as it results from two-photon excitation from the rovibronic ground state with linearly polarized radiation. When writing the vibronic transition matrix elements as complex numbers in polar form, ${ }^{27}$

$\left\langle n^{+} \Lambda^{+}=0, v^{+}\left|\mathrm{T}_{0}^{\prime k=0}\right| n^{\prime} \Lambda^{\prime}=0, v^{\prime}\right\rangle=\sqrt{C_{0}} \exp \left(i \phi_{0}\right)$,

$\left\langle n^{+} \Lambda^{+}=0, v^{+}\left|\mathrm{T}_{0}^{\prime k=2}\right| n^{\prime} \Lambda^{\prime}=0, v^{\prime}\right\rangle=\sqrt{C_{2}} \exp \left(i \phi_{2}\right)$,

with $C_{0}, C_{2} \in \mathbb{R}, C_{0}, C_{2} \geq 0$ and $\phi_{0}, \phi_{2} \in[0,2 \pi)$ and assuming the matrix elements with $k>2$ to vanish, evaluation of Eq. (37) yields

$$
\begin{aligned}
P_{\rho^{\prime}}\left(J^{\prime}=2, J^{+}=3 / 2\right)= & 0.13 C_{0}+0.07 C_{2} \\
& -0.11 \sqrt{C_{0} C_{2}} \cos \left(\phi_{0}-\phi_{2}\right)
\end{aligned}
$$

and

$$
\begin{aligned}
P_{\rho^{\prime}}\left(J^{\prime}=2, J^{+}=5 / 2\right)= & 0.20 C_{0}+0.10 C_{2} \\
& -0.16 \sqrt{C_{0} C_{2}} \cos \left(\phi_{0}-\phi_{2}\right) .
\end{aligned}
$$

The transition probability for ionization of an anisotropically populated level thus depends not only on the magnitude but also on the relative phase $\Delta \phi=\phi_{0}-\phi_{2}$ of the vibronic transition matrix elements. This effect is illustrated in Fig. 4. The relative strength of the ionization transitions may therefore in general not be calculated without information about these phases. $^{28}$

Owing to the non-vanishing cross terms, Eq. (37) may in general also not be substantially simplified. Provided the vibronic transition matrix elements are fully specified in terms of

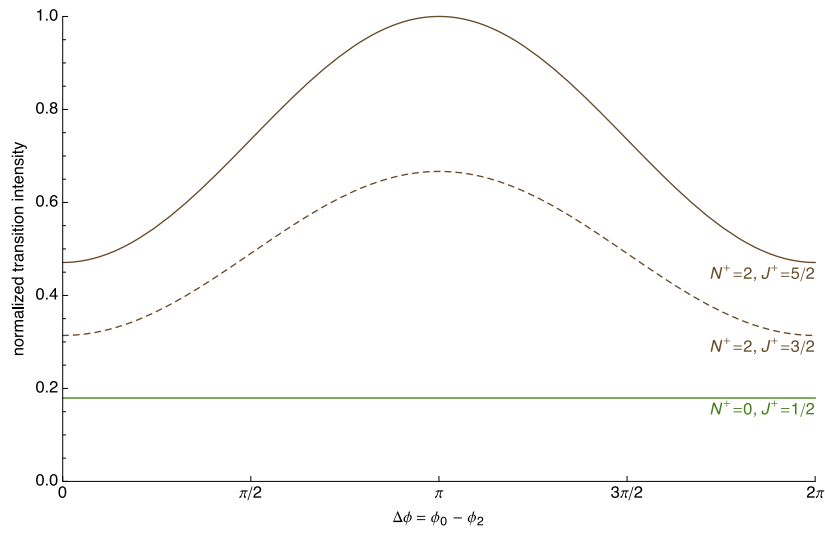

FIG. 4. Interference effects in photoionization of anisotropically populated states illustrated for the $N^{\prime}=2 \rightarrow N^{+}=2, J^{+}=5 / 2$ (solid, brown line) and the $N^{\prime}=2 \rightarrow N^{+}=2, J^{+}=3 / 2$ (dashed, brown line) transitions assuming the entire neutral population being confined to the $N^{\prime}=2, M_{N}^{\prime}=0$ Zeeman state. See text for details.

both their magnitude and phase (e.g., as a result of an ab initio calculation), calculation of the quantity $P_{\rho^{\prime}}\left(J^{\prime}, J^{+}\right)$is most conveniently achieved by evaluation of this equation with the help of a computer algebra system.

In many practically relevant cases, however, further simplification of Eq. (37) is possible. For photoionization by ejection of the photoelectron from a molecular orbital with predominantly s-type character (such as in $\mathrm{H}_{2},{ }^{29} \mathrm{~N}_{2},{ }^{30}$ or $\mathrm{O}_{2}{ }^{31}$ ), the ionization process is dominated by the vibronic transition matrix elements with the two lowest possible values for $k$, i.e., $k=0$ and $k=2$ for parity conserving transitions, while matrix elements with higher values of $k$ essentially vanish and may be neglected. Under these conditions, ionizing transitions with a change in the orbital-rotational angular momentum $(\Delta N \neq 0$, i.e., $\mathrm{O}(\Delta N=-2)$ and $\mathrm{S}(\Delta N=+2)$ lines $)$, may only occur due to the $k=2$ vibronic matrix element. Hence, only the $k=2$ term in Eq. (37) is relevant for these transitions. As a consequence, there are no cross terms occurring in that equation and hence also no phase dependencies are observed. This phase insensitivity is shown in Fig. 4 by the example of the $N^{\prime}=J^{\prime}=2 \rightarrow N^{+}=0, J^{+}=1 / 2$ transition.

Indeed, the S- and O-lines are relevant for the stateselective production of molecular cations by the method of threshold REMPI ${ }^{7-9}$ and we may thus treat these important cases even in the absence of information on the phases of the different vibronic matrix elements.

Evaluation of Eq. (37) for $\mathrm{S}$ and $\mathrm{O}$ lines, when taking into account the above-mentioned assumptions and approximations, yields

$$
\begin{aligned}
P_{\rho^{\prime}}^{(\mathrm{S}, \mathrm{O})}\left(J^{\prime}, J^{+}\right)= & 5\left(2 N^{\prime}+1\right)\left(2 N^{+}+1\right)\left(2 S^{\prime}+1\right)\left(2 J^{\prime}+1\right)\left(2 J^{+}+1\right) \sum_{l} \sum_{m_{l}} \sum_{m_{s}} \sum_{M_{J}^{\prime}, M_{J}^{+}} \rho^{\prime}\left(J^{\prime}, M_{J}^{\prime}\right) \\
& \times\left|\left\langle n^{+} \Lambda^{+}=0, v^{+}\left|\mathrm{T}_{0}^{\prime k=2}\right| n^{\prime} \Lambda^{\prime}=0, v^{\prime}\right\rangle\right|^{2}\left(\begin{array}{ccc}
l & 1 & 2 \\
-m_{l} & \mu_{0} & -p
\end{array}\right)^{2}\left(\begin{array}{ccc}
N^{+} & 2 & N^{\prime} \\
0 & 0 & 0
\end{array}\right)^{2} \\
& \times\left|\sum_{u=3 / 2}^{5 / 2}(2 u+1)\left(\begin{array}{ccc}
J^{+} & u & J^{\prime} \\
-M_{J}^{+} & w & M_{J}
\end{array}\right)\left(\begin{array}{ccc}
u & 2 & 1 / 2 \\
w & -p & m_{s}
\end{array}\right)\left(\begin{array}{ccc}
J^{+} & u & J^{\prime} \\
N^{+} & 2 & N^{\prime} \\
S^{+} & 1 / 2 & S^{\prime}
\end{array}\right)\right|^{2},
\end{aligned}
$$


where we have also used that $\Lambda^{+}=\Lambda=0$ for $\Sigma$ states.

\section{Hyperfine structure}

The effects discussed so far for ionizing transitions connecting spin-rotational levels are analogously found for hfs-resolved lines. The transition probability for photoionization of excited molecules with populations $\rho^{\prime}\left(F^{\prime}, M_{F}^{\prime}\right)$ for the different Zeeman states is given by

$$
\begin{aligned}
P_{\rho^{\prime}}\left(J^{\prime}, F^{\prime}, J^{+}, F^{+}\right)= & \sum_{l} \sum_{m_{l}} \sum_{m_{s}} \sum_{M_{F}^{\prime}, M_{F}^{+}} \rho^{\prime}\left(F^{\prime}, M_{F}^{\prime}\right) \mid\left(\left\langle n^{+} \Lambda^{+}, v^{+}, N^{+} \Lambda^{+} S^{+} J^{+} I^{+} F^{+} M_{F}^{+}\right|\left\langle s, m_{s}\right|\left\langle l, m_{l}\right|\right) \\
& \times\left.\mu\left|n^{\prime} \Lambda^{\prime}, v^{\prime}, N^{\prime} \Lambda^{\prime} S^{\prime} J^{\prime} I^{\prime} F^{\prime} M_{F}^{\prime}\right\rangle\right|^{2} .
\end{aligned}
$$

In a similar way as shown for Eq. (37) above, we obtain

$$
\begin{aligned}
P_{\rho^{\prime}}\left(J^{\prime}, F^{\prime}, J^{+}, F^{+}\right)= & \left(2 N^{\prime}+1\right)\left(2 N^{+}+1\right)\left(2 S^{\prime}+1\right)\left(2 J^{\prime}+1\right)\left(2 J^{+}+1\right)\left(2 F^{\prime}+1\right)\left(2 F^{+}+1\right) \delta_{I^{\prime} I^{+}} \\
& \times \sum_{l} \sum_{m_{l}} \sum_{m_{s}} \sum_{M_{F}^{\prime}, M_{F}^{+}} \rho^{\prime}\left(F^{\prime}, M_{F}^{\prime}\right) \mid \sum_{k=l \pm 1}(-1)^{k} \sqrt{2 k+1}\left(\begin{array}{ccc}
l & 1 & k \\
-m_{l} & \mu_{0} & -p
\end{array}\right)\left\langle n^{+} \Lambda^{+}, v^{+}\left|\mathrm{T}_{\Delta \Lambda}^{\prime_{\Lambda}}\right| n^{\prime} \Lambda^{\prime}, v^{\prime}\right\rangle \\
& \times\left(\begin{array}{ccc}
N^{+} & k & N^{\prime} \\
-\Lambda^{+} & \Delta \Lambda & \Lambda^{\prime}
\end{array}\right) \sum_{u=|k-s|}^{k+s}(-1)^{-u}(2 u+1)\left(\begin{array}{ccc}
u & k & s \\
w & -p & m_{s}
\end{array}\right)\left\{\begin{array}{ccc}
J^{+} & u & J^{\prime} \\
N^{+} & k & N^{\prime} \\
S^{+} & s & S^{\prime}
\end{array}\right)\left\{\begin{array}{ccc}
u & J^{\prime} & J^{+} \\
I^{\prime} & F^{+} & F^{\prime}
\end{array}\right\} \\
& \times\left.\left(\begin{array}{ccc}
F^{+} & u & F^{\prime} \\
-M_{F}^{+} & w & M_{F}^{\prime}
\end{array}\right)\right|^{2},
\end{aligned}
$$

upon substituting the results from Eqs. (29), (30), and (22) of Paper I. ${ }^{10}$ As before, evaluation of Eq. (43) requires in general both the magnitudes and the phases of the vibronic transition matrix elements. Hence, a significant "paper-and-pencil" simplification of Eq. (43) is not possible.

Once more, however, the experimentally interesting case of ionization out of predominantly s-type orbitals via $\mathrm{S}$ and $\mathrm{O}$ transitions can be treated even without concrete knowledge of the vibronic transition matrix elements. According to the reasoning above, Eq. (43) becomes for these transitions

$$
\begin{aligned}
P_{\rho^{\prime}}^{(\mathrm{S}, 0)}\left(J^{\prime}, F^{\prime}, J^{+}, F^{+}\right)= & 5\left(2 N^{\prime}+1\right)\left(2 N^{+}+1\right)\left(2 S^{\prime}+1\right)\left(2 J^{\prime}+1\right)\left(2 J^{+}+1\right)\left(2 F^{\prime}+1\right)\left(2 F^{+}+1\right) \delta_{I^{\prime} I^{+}} \\
& \times\left|\left\langle n^{+} \Lambda^{+}=0, v^{+}\left|\mathrm{T}_{0}^{k=2}\right| n^{\prime} \Lambda^{\prime}=0, v^{\prime}\right\rangle\right|^{2}\left(\begin{array}{ccc}
N^{+} & 2 & N^{\prime} \\
0 & 0 & 0
\end{array}\right)^{2} \sum_{l=1,3} \sum_{m_{l}} \sum_{m_{s}} \sum_{M_{F}^{\prime}, M_{F}^{+}} \rho^{\prime}\left(F^{\prime}, M_{F}^{\prime}\right) \\
& \times\left(\begin{array}{ccc}
l & 1 & 2 \\
-m_{l} & \mu_{0} & -p
\end{array}\right)^{2} \mid \sum_{u=3 / 2}^{5 / 2}(-1)^{-u}(2 u+1)\left(\begin{array}{ccc}
u & 2 & 1 / 2 \\
w & -p & m_{s}
\end{array}\right)\left\{\begin{array}{ccc}
J^{+} & u & J^{\prime} \\
N^{+} & 2 & N^{\prime} \\
S^{+} & 1 / 2 & S^{\prime}
\end{array}\right)\left\{\begin{array}{ccc}
u & J^{\prime} & J^{+} \\
I^{\prime} & F^{+} & F^{\prime}
\end{array}\right\} \\
& \times\left.\left(\begin{array}{ccc}
F^{+} & u & F^{\prime} \\
-M_{F}^{+} & w & M_{F}^{\prime}
\end{array}\right)\right|^{2} .
\end{aligned}
$$

Further simplification for a singlet neutral excited state $\left(S^{\prime}=0\right)$ and for linear polarized radiation $\left(\mu_{0}=0\right)$ yields

$$
\begin{aligned}
P_{\rho^{\prime}}^{\left(\mathrm{S}, \mathrm{O}, S^{\prime}=0, \text { lin. }\right)}\left(N^{\prime}, F^{\prime}, J^{+}, F^{+}\right)= & \frac{5}{2}\left(2 N^{+}+1\right)\left(2 N^{\prime}+1\right)\left(2 J^{+}+1\right)\left(2 F^{+}+1\right)\left(2 F^{\prime}+1\right) \delta_{I^{\prime} I^{+}}\left(\begin{array}{ccc}
N^{+} & 2 & N^{\prime} \\
0 & 0 & 0
\end{array}\right)^{2} \\
& \times\left|\left\langle n^{+} \Lambda^{+}=0, v^{+}\left|\mathrm{T}_{0}^{\prime k=2}\right| n^{\prime} \Lambda^{\prime}=0, v^{\prime}\right\rangle\right|^{2} \sum_{l=1,3} \sum_{m_{l}} \sum_{m_{s}} \sum_{M_{F}^{\prime}, M_{F}^{+}} \rho^{\prime}\left(F^{\prime}, M_{F}^{\prime}\right) \\
& \times\left(\begin{array}{ccc}
l & 1 & 2 \\
-m_{l} & 0 & m_{l}
\end{array}\right) \mid \sum_{u=3 / 2}^{2}(2 u+1)\left(\begin{array}{ccc}
u & 2 & 1 / 2 \\
-m_{l}-m_{S} & m_{l} & m_{s}
\end{array}\right)\left\{\begin{array}{ccc}
J^{+} & u & N^{\prime} \\
2 & N^{+} & 1 / 2
\end{array}\right\} \\
& \times\left.\left\{\begin{array}{ccc}
u & N^{\prime} & J^{+} \\
I^{\prime} & F^{+} & F^{\prime}
\end{array}\right\}\left(\begin{array}{ccc}
F^{+} & u & F^{\prime} \\
-M_{F}^{+} & -m_{l}-m_{S} & M_{F}^{\prime}
\end{array}\right)\right|^{2} .
\end{aligned}
$$




\section{THE $\left[2+1^{\prime}\right]$ REMPI PROCESS}

Having discussed both the excitation as well as the ionization step, we may now combine the results from Secs. III and IV to a model for the $\left[2+1^{\prime}\right]$ REMPI process.

The populations $\rho^{\prime}\left(F^{\prime}, M_{F}^{\prime}\right)$ of the hfs levels in the neutral excited state generated in the REMPI process are proportional to the excitation rates $R_{F^{\prime \prime} \rightarrow F^{\prime}, M_{F}^{\prime}}$ of the transitions populating these levels multiplied with the population in the relevant levels of the neutral vibronic ground state $\rho^{\prime \prime}\left(F^{\prime \prime}\right)$,

$$
\rho^{\prime}\left(F^{\prime}, M_{F}^{\prime}\right) \propto \sum_{F^{\prime \prime}=\left|J^{\prime \prime}-I^{\prime \prime}\right|}^{J^{\prime \prime}+I^{\prime \prime}} R_{F^{\prime \prime} \rightarrow F^{\prime}, M_{F}^{\prime}} \rho^{\prime \prime}\left(F^{\prime}\right) .
$$

Here, the sum over all the ground-state hfs levels has been included since hyperfine structure is supposed to be unresolved in the two-photon excitation step. The relative hfs populations of the electronic-ground-state molecules $\mathrm{AB}$ are given by a Boltzmann distribution,

$$
\rho^{\prime \prime}\left(F^{\prime \prime}\right)=g_{F^{\prime \prime}} \exp \left(-E_{F^{\prime \prime}} / k_{\mathrm{B}} T\right),
$$

with $E_{F^{\prime \prime}}$ the energy of the level $F^{\prime \prime}$ and $g_{F^{\prime \prime}}$ its degeneracy, $k_{\mathrm{B}}$ the Boltzmann constant, and the temperature $T$. Since the hfs splittings are usually small compared to the thermal energies $\left(\Delta E_{\mathrm{hfs}} \ll k_{\mathrm{B}} T\right)$, the energies $E_{F^{\prime \prime}}$ are essentially equal for all hfs levels of one rotational level and the exponential factor in Eq. (47) is nearly identical for them. The relative hfs populations within the same rotational level are thus approximately given by the degeneracy of the hfs levels: $\rho^{\prime \prime}\left(F^{\prime \prime}\right) \propto g_{F^{\prime \prime}}$. The excitation rate $R_{F^{\prime \prime} \rightarrow F^{\prime}, M_{F}^{\prime}}$, on the other hand, is proportional to the two-photon line strength worked out in Sec. III normalized by the respective ground-state degeneracy $g_{F^{\prime \prime}}$,

$$
R_{F^{\prime \prime} \rightarrow F^{\prime}, M_{F}^{\prime}} \propto 1 / g_{F^{\prime \prime}} \times S_{\mathrm{S}, \mathrm{O}}^{(\text {lin })}\left(F^{\prime \prime}, F^{\prime}, M_{F}^{\prime}\right),
$$

where $S_{\mathrm{S}, \mathrm{O}}^{(\text {lin) }}\left(F^{\prime \prime}, F^{\prime}, M_{F}^{\prime}\right)$ is given by Eq. (35) (without loss of generality, we restrict ourselves to $\mathrm{S}, \mathrm{O}$ transitions here). Combining the results of Eqs. (46)-(48), we thus obtain the relative hfs populations of the neutral, excited molecules $\mathrm{AB}^{*}$ as

$$
\rho^{\prime}\left(F^{\prime}, M_{F}^{\prime}\right) \propto \sum_{F^{\prime \prime}=\left|J^{\prime \prime}-I^{\prime \prime}\right|}^{J^{\prime \prime}+I^{\prime \prime}} S_{\mathrm{S}, \mathrm{O}}^{(\mathrm{iin})}\left(F^{\prime \prime}, F^{\prime}, M_{F}^{\prime}\right) .
$$

To obtain the relative populations $\rho^{+}\left(J^{+}, F^{+}\right)$of the molecular ions $\mathrm{AB}^{+}$, we substitute the neutral excited state populations $\rho^{\prime}\left(F^{\prime}, M_{F}^{\prime}\right)$ in Eq. (44) (or Eq. (45) for a singlet neutral state) by the expression of Eq. (49), i.e.,

$$
\rho_{F^{\prime}}^{+}\left(J^{+}, F^{+}\right) \propto P_{\rho^{\prime}\left(F^{\prime}, M_{F}^{\prime}\right)^{\prime}}^{(\mathrm{S}, \mathrm{O})}\left(J^{\prime}, F^{\prime}, J^{+}, F^{+}\right) .
$$

Here, the subscript $F^{\prime}$ has been added indicating that this quantity refers to the ionic population generated via ionization from a particular $F^{\prime}$ hyperfine level of the neutral excited state. If the hyperfine structure is not resolved in the ionization step, the total ionic population in a particular ionic hyperfine level $F^{+}$is given by the sum over all neutral, excited $\mathrm{AB}^{*}$ hfs levels $F^{\prime}$,

$$
\rho_{\mathrm{tot}}^{+}\left(J^{+}, F^{+}\right)=\sum_{F^{\prime}} \rho_{F^{\prime}}^{+}\left(J^{+}, F^{+}\right)
$$

In REMPI experiments, often the two planes of polarization of the excitation and the ionization laser are not parallel, but tilted by some angle $\alpha$ relative to each other (e.g., as a consequence of the geometry of frequency multiplication stages used for UV generation). If so, the expressions given above for the excitation and the ionization step are referring to two different space-fixed frames. ${ }^{32}$ Such a tilting between the two polarization vectors may be taken into account by multiplication of the populations calculated in the excitation frame (labeled below by projection quantum numbers $\bar{M}_{F}^{\prime}$ as arguments) with a squared Wigner rotation matrix element $\mathcal{D}_{\bar{M}_{F}^{\prime}}^{F_{F} M_{F}^{\prime}}(0, \alpha, 0)$ and summing over the projection quantum numbers in the excitation frame $\bar{M}_{F}^{\prime} 11,12$

$$
\begin{aligned}
\rho_{\alpha}^{\prime}\left(F^{\prime}, M_{F}^{\prime}\right)= & \sum_{\bar{M}_{F}^{\prime}=-F^{\prime}}^{F^{\prime}}\left[\mathcal{D}_{\bar{M}_{F}^{\prime} M_{F}^{\prime}}^{\prime}(0, \alpha, 0)\right]^{2} \rho^{\prime}\left(F^{\prime}, \bar{M}_{F}^{\prime}\right) \\
\propto & \sum_{\bar{M}_{F}^{\prime}=-F^{\prime}}^{F^{\prime}}\left[\mathcal{D}_{\bar{M}_{F}^{\prime} M_{F}^{\prime}}^{\prime}(0, \alpha, 0)\right]^{2} \\
& \times \sum_{F^{\prime \prime}=\left|J^{\prime \prime}-I^{\prime \prime}\right|}^{J^{\prime \prime}+I^{\prime \prime}} S_{\mathrm{S}, \mathrm{O}}^{\text {(lin. pol. })}\left(F^{\prime \prime}, F^{\prime}, \bar{M}_{F}^{\prime}\right) .
\end{aligned}
$$

The populations $\rho_{\alpha}^{\prime}\left(F^{\prime}, M_{F}^{\prime}\right)$ are then substituted into Eq. (50) instead of those obtained from Eq. (49) yielding

$$
\rho_{F^{\prime}, \alpha}^{+}\left(J^{+}, F^{+}\right) \propto P_{\rho_{\alpha}^{\prime}\left(F^{\prime}, M_{F}^{\prime}\right)}^{(\mathrm{S}, \mathrm{O})}\left(J^{\prime}, F^{\prime}, J^{+}, F^{+}\right)
$$

and

$$
\rho_{\text {tot, } \alpha}^{+}\left(J^{+}, F^{+}\right)=\sum_{F^{\prime}} \rho_{F^{\prime}, \alpha}^{+}\left(J^{+}, F^{+}\right) .
$$

Although we have concentrated here on the $\left[2+1^{\prime}\right]$ REMPI scheme, our calculations may be adapted for other two-color REMPI schemes as well. In the case of $\left[1+1^{\prime}\right]$ REMPI, the formulae for the excitation step derived in Sec. III and then employed in Sec. V must be replaced by the corresponding well-known expressions for one-photon transitions (see, e.g., Refs. 33 and 34 and references therein), for $\left[n+1^{\prime}\right]$ REMPI (with $n>2$ ) the excitation step may be treated according to the theory of multiphoton transitions in diatomic molecules discussed in Refs. 14, 35, and 36, where also the corresponding formulae for the Hund's case (a) angular momentum coupling scheme as well as for intermediate Hund's case (a)-(b) coupling situations are found. Similarly, the expressions given here for the ionization step may be adapted for other angular momentum coupling cases by means of a suitable basis transformations as, e.g., outlined in Ref. 33. 


\section{APPLICATION: HYPERFINE POPULATIONS OF MOLECULAR NITROGEN IONS PRODUCED BY $\left[2+1^{\prime}\right]$ REMPI}

\section{A. Non-hfs-resolved photoionization of molecular nitrogen}

\section{1. $S(0)-O(2)$ REMPI scheme}

As a first application of our model, we study the REMPI scheme previously used for the rotationally state-selective production of $\mathrm{N}_{2}^{+}$ions via excitation of the $\mathrm{N}_{2} \mathrm{a}^{\prime \prime}{ }^{1} \Sigma_{\mathrm{g}}^{+}$state. , $^{5,8,9}$ Here, we are interested in the relative hfs populations of $\mathrm{N}_{2}^{+}$ions produced in the rovibrational ground state by the REMPI sequence $N^{\prime \prime}=0 \rightarrow N^{\prime}=2 \rightarrow N^{+}=0$ for the $I=2$ nuclear spin manifold of $\mathrm{N}_{2} / \mathrm{N}_{2}^{+}$. The energy levels and corresponding Zeeman states involved are shown in Fig. 5. The hyperfine structure is supposed to be unresolved in both the excitation and the ionization step. Hence, we calculate the ionic populations using Eqs. (51) and (55). As an example of a corresponding calculation without treatment of hfs, see, e.g., Ref. 37.

The relative populations in the neutral, excited $\mathrm{N}_{2} \mathrm{a}^{\prime \prime}{ }^{1} \Sigma_{\mathrm{g}}^{+}$ state are shown in Fig. 6. In the left column of the figure, the populations are shown with reference to the excitation frame. In the right column, the corresponding values after transformation to the ionization frame are given. The effect that only a subset of the Zeeman states may be populated by excitation with linear polarized radiation is clearly visible in the left-hand column of Fig. 6: Zeeman states with $\bar{M}_{F}^{\prime}>2$ are not populated due to the selection rule $\bar{M}_{F}^{\prime}=M_{F}^{\prime \prime}$ for excitation with linear polarized radiation (polarization vector parallel to quantization axis).

For the right column of Fig. 6, an angle of $\alpha=90^{\circ}$ between the two polarization vectors of excitation and ionization has been assumed. The frame transformation described by Eq. (52) leads to a redistribution of the population such that Zeeman states not populated in the excitation frame, are populated with respect to the ionization frame of reference.

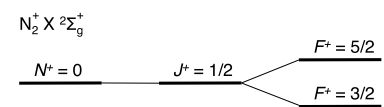

$$
\begin{aligned}
& -5 / 2 \quad-3 / 2 \quad-1 / 2 \quad 1 / 2 \quad 3 / 2 \quad 5 / 2 \\
& -3 / 2-1 / 2 \quad 1 / 2 \quad 3 / 2
\end{aligned}
$$

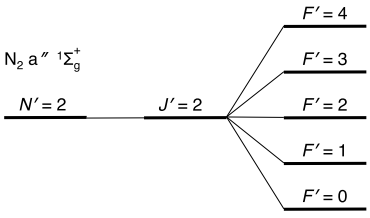

$$
\begin{aligned}
& -4-\frac{-3}{-2}-\frac{-1}{0} \div-1 \frac{2}{2} \stackrel{3}{4} \\
& -3-2-1 \leq 0-12 \\
& -2-1 \div 12 \\
& -1 \pm 1 \\
& \text { 음 }
\end{aligned}
$$

$$
\mathrm{N}_{2} \mathrm{X}^{1} \Sigma_{\mathrm{g}}^{+}
$$

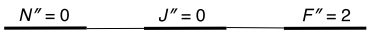

$$
-2-1 ㅇ ㅡ
$$

FIG. 5. Level diagram for the $\mathrm{S}(0)-\mathrm{O}(2)$ REMPI of $\mathrm{N}_{2}$ showing the relevant fs and hfs levels as well as the Zeeman states for the REMPI sequence $N^{\prime \prime}=0 \rightarrow N^{\prime}=2 \rightarrow N^{+}=0$ of the $I=2$ nuclear spin manifold of $\mathrm{N}_{2} / \mathrm{N}_{2}^{+}$. (As the exact hyperfine structure of the $\mathrm{a}^{\prime \prime}{ }^{1} \Sigma_{\mathrm{g}}^{+}$state is unknown, levels of this state are ordered by their degeneracy.)

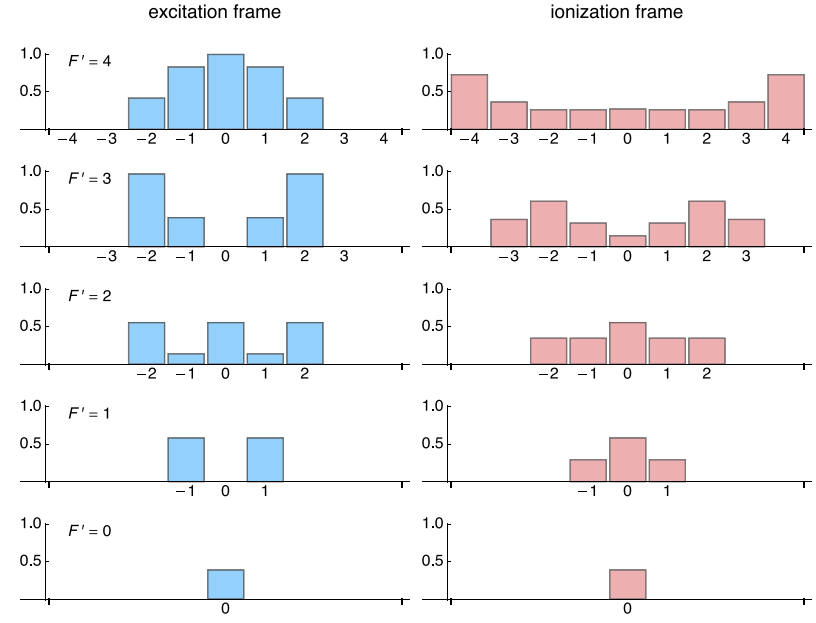

FIG. 6. $\mathrm{S}(0)-\mathrm{O}(2)$ REMPI scheme of $\mathrm{N}_{2}$ : bar charts of the relative populations in the $\mathrm{a}^{\prime \prime} \Sigma_{\mathrm{g}}^{+}$neutral excited state for the REMPI sequence $N^{\prime \prime}=0 \rightarrow N^{\prime}=2 \rightarrow N^{+}=0$. The charts in the left column show the relative populations in the Zeeman states of the hfs levels with $F^{\prime}=4$ to 0 (top to bottom) with respect to the excitation frame of reference. In the right column, the same populations are shown in the ionization frame of reference, when assuming an angle of $\alpha=90^{\circ}$ between the polarization vectors of the excitation and ionization laser beams. The bars are labeled by the projection angular momentum quantum numbers below the horizontal axis.

The relative ionic populations of the different $\mathrm{N}_{2}^{+}$hfs levels produced in this REMPI scheme are shown in Fig. 7 as blue and red bars for parallel $\left(\alpha=0^{\circ}\right)$ and perpendicular $\left(\alpha=90^{\circ}\right)$ polarization vectors, respectively.

For reference, the white, dashed-edged bars show the relative populations when assuming them to be proportional to the degeneracy of the levels. We refer to them as the "pseudo-thermal" populations, as these are the relative hfs populations of a thermal ensemble in the limit of the thermal energy $k_{\mathrm{B}} T$ being large compared to the hfs splittings.

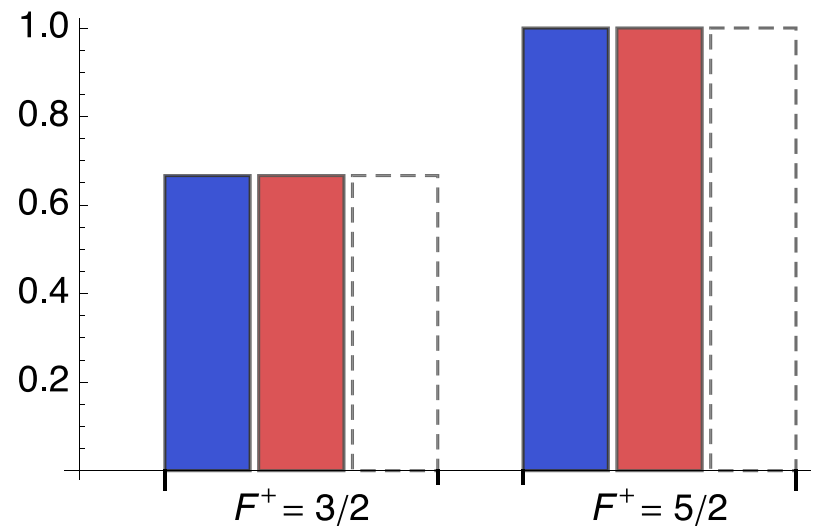

FIG. 7. Populations in the rovibrational ground state of $\mathrm{N}_{2}^{+}$produced by $\mathrm{S}(0)-\mathrm{O}(2)$ REMPI: relative populations of the two hfs levels of $\mathrm{N}_{2}^{+}$ions produced by the REMPI sequence $N^{\prime \prime}=0 \rightarrow N^{\prime}=2 \rightarrow N^{+}=0$. The blue bars show the populations as obtained for parallel polarization vectors for excitation and ionization $\left(\alpha=0^{\circ}\right)$, for the red ones an angle of $\alpha=90^{\circ}$ between the two polarization vectors is assumed. For reference, the populations expected from the "pseudo-thermal" model (see text) are indicated by the white, dashed-edged bars. (Values are normalized to yield equal total populations for parallel and perpendicular polarization, as well as for the "pseudo-thermal" model, then normalized to unity for the highest value.) 


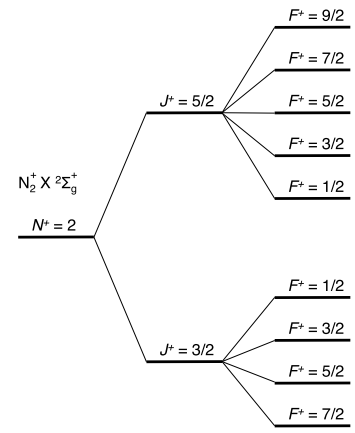

$$
\begin{aligned}
& -9 / 2 \quad-7 / 2 \quad-5 / 2 \quad-3 / 2 \quad-1 / 2 \quad 1 / 2 \quad 3 / 2 \quad 5 / 2 \quad 7 / 2 \quad 9 / 2 \\
& -7 / 2-5 / 2 \quad-3 / 2 \quad-1 / 2 \quad 1 / 2 \quad 3 / 2 \quad 5 / 2 \quad 7 / 2 \\
& -5 / 2-3 / 2-1 / 2 \quad 1 / 2 \quad 3 / 2 \quad 5 / 2 \\
& -3 / 2-1 / 2 \quad 1 / 2 \\
& -1 / 21 / 2 \\
& -1 / 2 \quad 1 / 2 \\
& -3 / 2-1 / 2 \quad 1 / 2 \quad 3 / 2 \\
& -5 / 2 \quad-3 / 2 \quad-1 / 2 \text { 1/2 } 3 / 2 \quad 5 / 2
\end{aligned}
$$

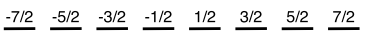

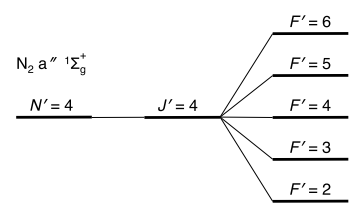

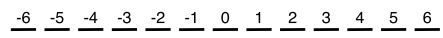

$$
\begin{aligned}
& -5-4-\frac{-3}{-2}-2-1
\end{aligned}
$$

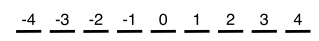

$$
\begin{aligned}
& -3-2-1 \\
& \underline{-2}-1 \div
\end{aligned}
$$

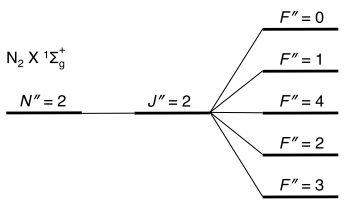

$$
\begin{aligned}
& \stackrel{0}{0} \\
& -1 \div \\
& -4-3 \frac{-2}{-1} \div 0 \frac{1}{2} \stackrel{3}{4} \\
& \text {-2 - }-1 \text { 은 } \\
& \text {-3 }-2-\frac{-1}{0} 1 ㅡ
\end{aligned}
$$

FIG. 8. Level diagram for the $\mathrm{S}(2)-\mathrm{O}(4)$ REMPI scheme of $\mathrm{N}_{2}$ showing the relevant fs and hfs levels as well as the Zeeman states for the REMPI sequence $N^{\prime \prime}=2 \rightarrow N^{\prime}=4 \rightarrow N^{+}=2$ of the $I=2$ nuclear spin manifold of $\mathrm{N}_{2} / \mathrm{N}_{2}^{+}$. (The energetic order of the hfs levels in the neutral $\mathrm{N}_{2}{ }^{1} \Sigma_{\mathrm{g}}^{+}$state has been estimated using electric-quadrupole coupling constants extrapolated from spectroscopic data on the neutral $\mathrm{N}_{2} \mathrm{~A}^{3} \Sigma_{\mathrm{u}}^{+}$state and from $\mathrm{N}_{2}$ complexes. ${ }^{38-40}$ The hfs levels of the $\mathrm{N}_{2} \mathrm{a}^{\prime \prime} \Sigma_{\mathrm{g}}^{+}$are ordered by their degeneracies.)

In this particular case, the naïve pseudo-thermal model yields the same relative populations as our ionization model does. Moreover, the relative populations predicted by our model are identical for parallel and perpendicular polarization vectors. As shown below, these coincidences are particular for the $\mathrm{S}(0)-\mathrm{O}(2)$ ionization sequence and do in general not occur.

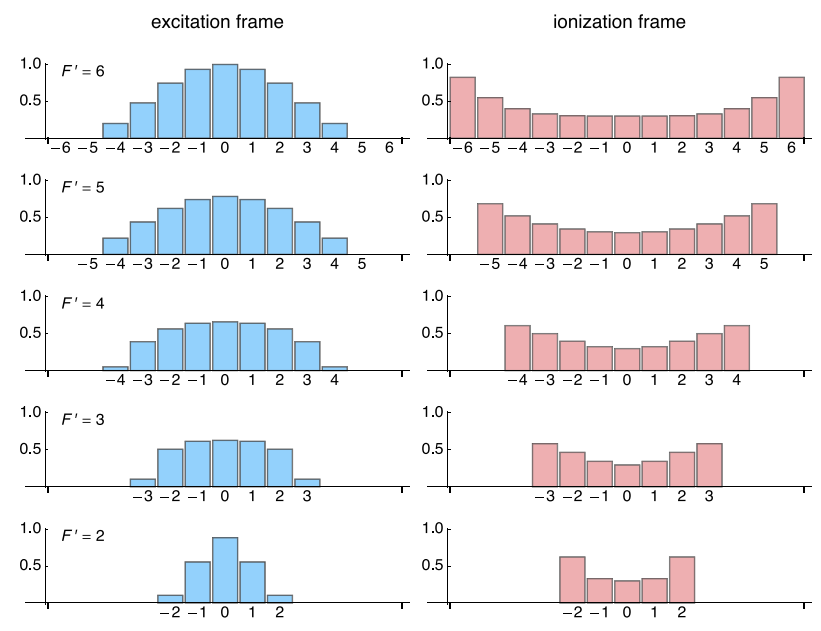

FIG. 9. $\mathrm{S}(2)-\mathrm{O}(4)$ REMPI scheme of $\mathrm{N}_{2}^{+}$: bar charts of the relative populations in the $\mathrm{a}^{\prime \prime}{ }^{1} \Sigma_{\mathrm{g}}^{+}$neutral excited state for the REMPI sequence $N^{\prime \prime}=2$ $\rightarrow N^{\prime}=4 \rightarrow N^{+}=2$. The charts in the left column show the relative populations in the Zeeman states of the hfs levels with $F^{\prime}=6$ to 2 (top to bottom) with respect to the excitation frame of reference. In the right column, the same populations are shown in the ionization frame of reference, when assuming an angle of $\alpha=90^{\circ}$ between the polarization vectors of excitation and ionization. The bars are labeled by the projection angular momentum quantum numbers below the horizontal axis.

\section{S(2)-O(4) REMPI scheme}

As a more complex example, we analyze the REMPI of $\mathrm{N}_{2}$ via the $\mathrm{a}^{\prime \prime}{ }^{1} \Sigma_{\mathrm{g}}^{+}$state through the sequence $N^{\prime \prime}=2 \rightarrow N^{\prime}$ $=4 \rightarrow N^{+}=2$. The relevant energy levels and Zeeman states are depicted in Fig. 8.

The populations in the neutral excited $\mathrm{a}^{\prime \prime}{ }^{1} \Sigma_{\mathrm{g}}^{+}$and the ionic $\mathrm{X}^{2} \Sigma_{\mathrm{g}}^{+}$state are shown in Figs. 9 and 10, respectively. Like in the previous example, the left column of Fig. 9 shows the populations in the Zeeman states belonging to the hfs levels of the $\mathrm{a}^{\prime \prime}{ }^{1} \Sigma_{\mathrm{g}}^{+}$state with respect to the excitation frame of reference, whereas the right column shows them with respect to the ionization frame. As before, an angle of $\alpha=90^{\circ}$ is assumed between the two polarization vectors. Once more, the effect of diminished populations in Zeeman states with high absolute values for the projection angular momentum quantum number due to the selection rules for the excitation step is observed. Also, the redistribution of
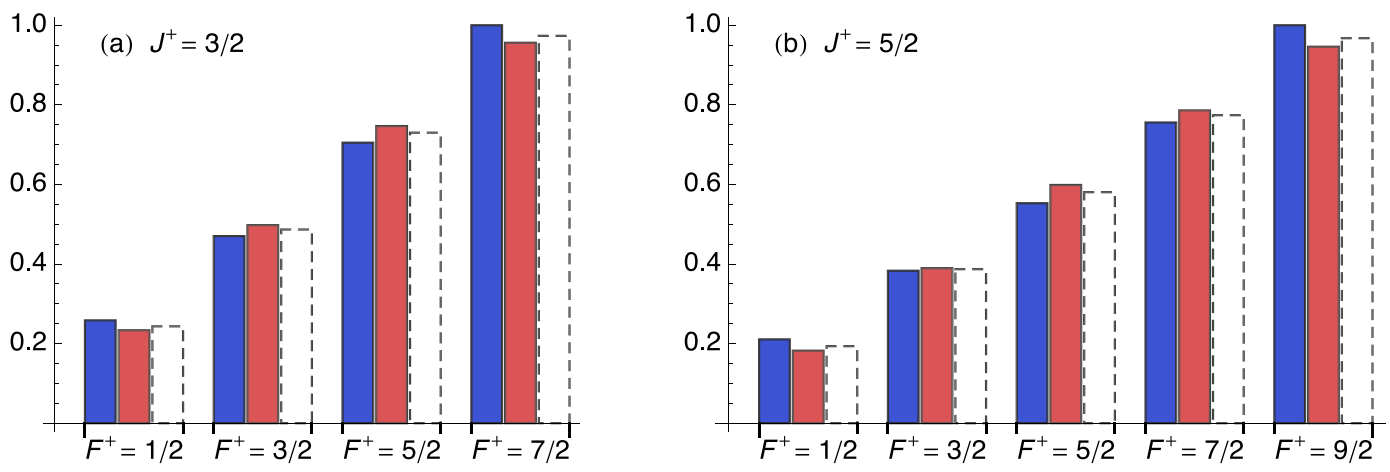

FIG. 10. Ionic populations due to $\mathrm{S}(2)-\mathrm{O}(4)$ REMPI of $\mathrm{N}_{2}^{+}$: relative populations of the fs and hfs levels of the $N^{+}=2$ rotational state of $\mathrm{N}_{2}^{+}$produced by the REMPI sequence $N^{\prime \prime}=2 \rightarrow N^{\prime}=4 \rightarrow N^{+}=2$. (a) values for the $J^{+}=3 / 2$ fine structure level, (b) corresponding values for the $J^{+}=5 / 2$ spin-rotation component. The blue bars show the populations obtained for parallel polarization vectors for excitation and ionization $\left(\alpha=0^{\circ}\right)$, for the red ones an angle of $\alpha=90^{\circ}$ between the two polarization vectors has been assumed. For reference, the populations expected from the pseudo-thermal model (see text) are indicated by the white, dashed-edged bars. 
(a) $\alpha=0^{\circ}$

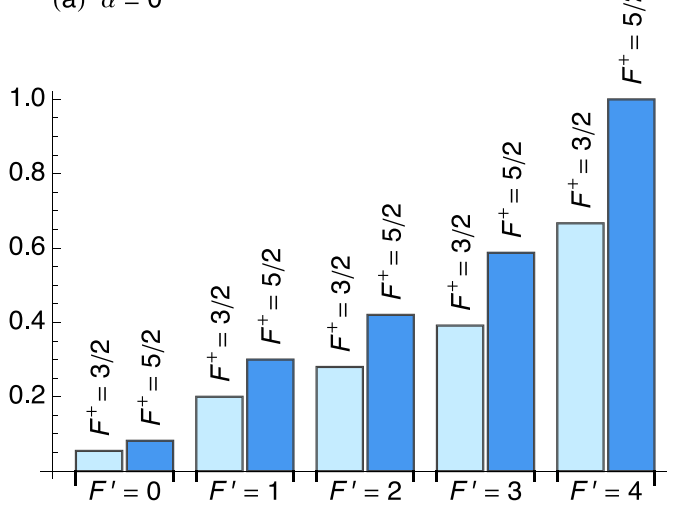

(b) $\alpha=90^{\circ}$

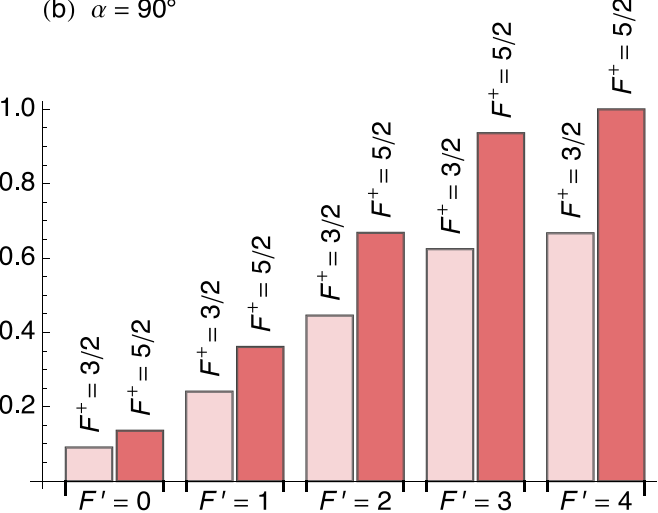

FIG. 11. Populations in the rovibrational ground state of $\mathrm{N}_{2}^{+}$due to $\mathrm{S}(0)-\mathrm{O}(2) \mathrm{REMPI}$ for specific ionizing hfs transitions: relative contributions to the two ionic hfs populations $F^{+}=3 / 2$ and $F^{+}=5 / 2$ from individual hfs levels of the neutral $\mathrm{a}^{\prime \prime}{ }^{1} \Sigma_{\mathrm{g}}^{+}$state $\left(F^{\prime}=0, \ldots, 4\right)$ for parallel (a) and perpendicular (b) polarization vectors. Ionization from all neutral hfs levels populates both ionic hfs states, no clear propensity is observed.

population in course of the frame transformation described by Eq. (52) is seen again. The relative hfs populations in the $J^{+}=3 / 2$ and $J^{+}=5 / 2$ spin-rotational levels of the $\mathrm{N}_{2}^{+}$ ion are shown in Figs. 10(a) and 10(b), respectively. The populations are shown for both parallel (blue bars) and perpendicular (red bars) polarization vectors for ionization and excitation. For comparison, also the pseudo-thermal populations are indicated (white, dashed-edged bars). In contrast to the previous example, the relative hfs populations obtained by our REMPI model now deviate from the pseudothermal populations. As these deviations are small, however, they might only have a minor effect on experiments with molecular $\mathrm{N}_{2}^{+}$ions produced by this and similar REMPI schemes. We note that the Hund's case $\left(b_{\beta_{J}}\right)$ basis states in rotational excited states of $\mathrm{N}_{2}^{+}$are mixed by off-diagonal terms in the hfs Hamiltonian leading to a mixing of the two fine structure components. ${ }^{6,23,41}$ Since we have found this mixing to only have a minor effect on the hfs populations predicted by our model, ${ }^{23}$ we have neglected it here.

\section{B. Hfs-resolved photoionization of molecular nitrogen}

So far, we have analyzed hfs-state populations of $\mathrm{N}_{2}^{+}$ions generated by hfs-unresolved photoionization. This means, ions were assumed to have been produced in a REMPI process, in which the hyperfine structure is not resolved, but the ionic populations were then supposed to be probed in a hfs-resolved manner, such as by hfs-resolved vibrational spectroscopy of the cation. ${ }^{6}$
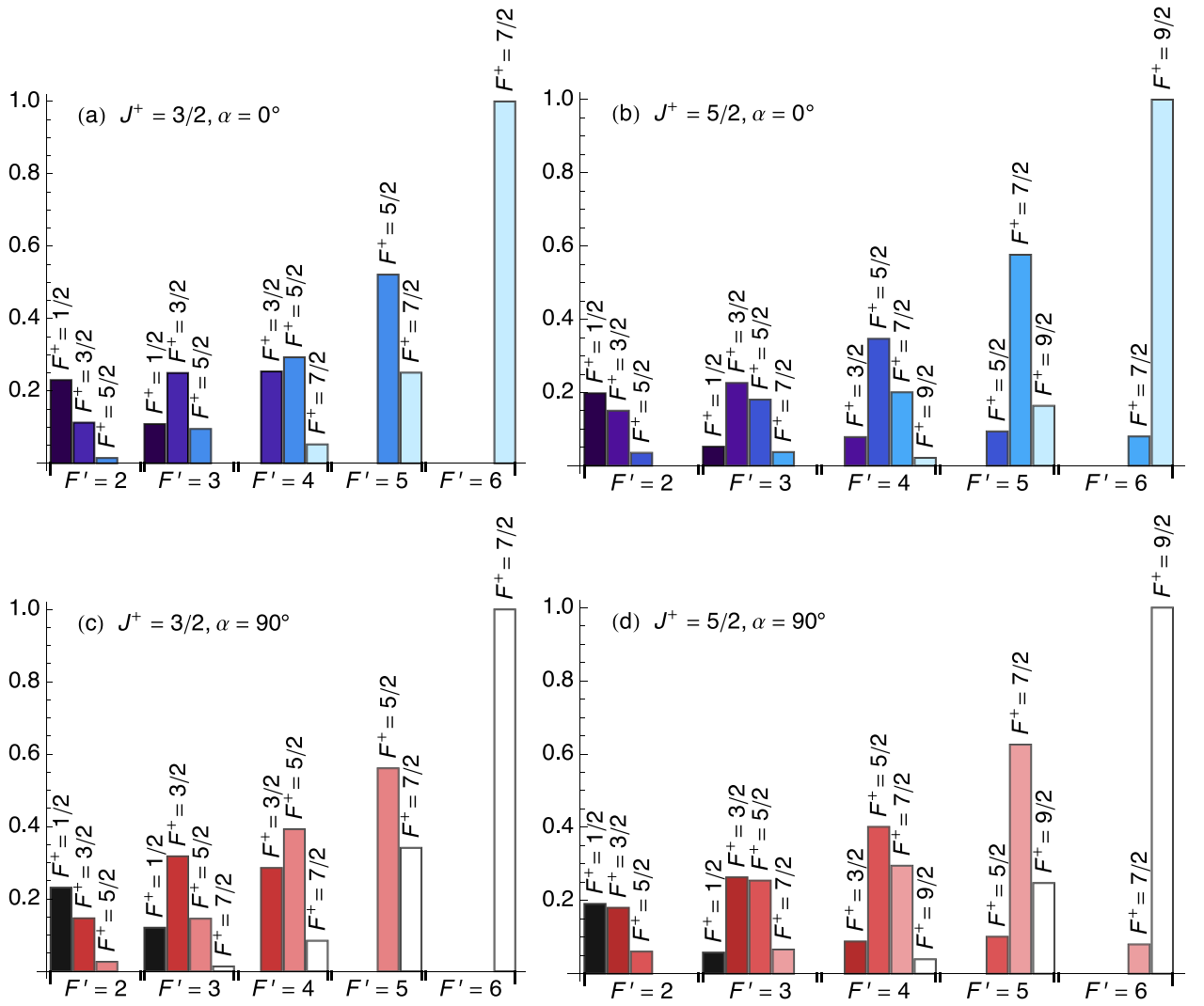

(d) $J^{+}=5 / 2, \alpha=90^{\circ}$

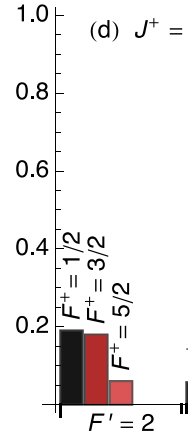

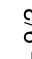

a

立
FIG. 12. Ionic populations due to $\mathrm{S}(2)$ $\mathrm{O}(4)$ REMPI of $\mathrm{N}_{2}^{+}$for specific ionization hfs transitions. Upper row: contributions to the relative hfs populations of $\mathrm{N}_{2}^{+}$ions for the $J^{+}=3 / 2$ (panel (a)) and $J^{+}=5 / 2$ (panel (b)) spin-rotation components from individual hfs levels of the neutral $\mathrm{a}^{\prime \prime}{ }^{1} \Sigma_{\mathrm{g}}^{+}$state assuming parallel polarization vectors of excitation and ionization $\left(\alpha=0^{\circ}\right)$. Lower row: corresponding values for perpendicular polarization vectors $\left(\alpha=90^{\circ}\right)$. For most transitions, a distinct propensity is observed: Ionization from a particular neutral hfs level $\left(F^{\prime}=2, \ldots, 4\right)$ preferentially results in a population in only one, eventually two, ionic hfs level. (Values $<10^{-2}$ have been suppressed for clarity.) 
Since selectivity over the vibronic and spin-rotational degrees of freedom in the REMPI process has already been achieved, ${ }^{8,9}$ extending this selectivity to the hfs domain, i.e., producing molecular ions also in a hfsstate-selective manner, is appealing-particularly in view of emerging non-destructive and coherent techniques in molecular spectroscopy. ${ }^{23,42,43}$

Here, we study the implications of our REMPI model for such a hfs-state-selective preparation scheme by analyzing the relative populations of $\mathrm{N}_{2}^{+}$ions for hfs-resolved ionization transitions. This means, we suppose the same neutral, excited populations as before (Figs. 6 and 9), but calculate the relative ionic populations for particular $F^{\prime} \rightarrow F^{+}$transitions using Eqs. (50) and (54). We are interested in possible propensities for these hfs-resolved ionization transitions, as these could enable achieving state-selectivity.

The results obtained for the $\mathrm{S}(0)-\mathrm{O}(2)$ REMPI sequence are shown in Fig. 11. As seen from this figure, ionization from all hfs levels of the neutral excited $\mathrm{N}_{2} \mathrm{a}^{\prime \prime}{ }^{1} \Sigma_{\mathrm{g}}^{+}$state leads to ionic populations in both hfs levels of the rovibronic ground state of $\mathrm{N}_{2}^{+}$. In other words, no clear propensity is observed.

Hence, hfs-state-selective production of $\mathrm{N}_{2}^{+}$in the rovibronic ground state would have to be achieved almost entirely by spectroscopic addressing, e.g., by spectroscopically resolving all accessible ionization thresholds and ionizing selectively above the lowest one. ${ }^{8,9}$ Whether this is possible, depends on the bandwidth of the radiation used for ionization and the hfs splitting in the $\mathrm{N}_{2} \mathrm{a}^{\prime \prime}{ }^{1} \Sigma_{\mathrm{g}}^{+}$state. The latter is unknown at present, since the spectroscopic investigation of this state ${ }^{44-47}$ has not yet achieved hyperfine resolution.

The hfs-resolved results from our model for the $\mathrm{S}(2)-\mathrm{O}(4)$ REMPI scheme are shown in Fig. $12 .^{48}$ For the $\mathrm{N}_{2}^{+}$ions with $N^{+}=2$ produced in this scheme, the relative populations exhibit a pattern remarkably different from that seen in the previous example. Ionization via certain hfs levels of the neutral excited a" ${ }^{1} \Sigma_{\mathrm{g}}^{+}$state populate almost exclusively particular hfs levels in the $\mathrm{N}_{2}^{+}$ion. In other words, clear propensities are observed. For the majority of the transitions, these characteristics are summarized by the propensity rule $\Delta J=\Delta F$ (with $\Delta J=J^{+}-N^{\prime}$ and $\Delta F=F^{+}-F^{\prime}$ ). Deviations from this rule are observed for $J^{+}=3 / 2$ at low values of $F^{\prime}$. We note that the effects of changing the polarization geometry are only slight in this case.

As a consequence of this propensity, hfs-state-selectivity can be achieved even without full spectroscopic resolution of individual hfs transitions in the ionization step.

\section{SUMMARY AND CONCLUSIONS}

In this paper, we have presented a model for the calculation of the relative populations of fine and hyperfine levels of molecular cations produced by resonance-enhanced multiphoton ionization (REMPI). Our model is based on understanding the REMPI process as two separate steps, a bound-bound neutral-ground-to-neutral-excited-state transition followed by the ionizing transition generating the cation.
Compared to the model for fine- and hyperfine structure effects in one-photon ionization presented in Paper $\mathrm{I},{ }^{10}$ description of the REMPI process requires considering two additional effects: hyperfine effects in the neutral bound-bound multiphoton transition and ionization of an anisotropic sample of neutral molecules.

Anisotropy complicates the calculation of transition probabilities and-in general-leads to interference effects between different vibronic transition matrix elements, resulting in a dependence of the observed ionization intensities not only on the magnitudes but also on the phases of these matrix elements. Hence, the prediction of transition intensities is in general only possible with vibronic transition matrix elements fully characterized by both their magnitude and their phase. However, in the practically relevant cases of $\mathrm{S}$ and $\mathrm{O}$ ionization transitions with the photoelectron ejected from a molecular orbital with predominantly s-type character, calculation of the relative populations of fine and hyperfine levels in the cation is possible without such detailed information.

We have shown the implications of our model using the REMPI of molecular nitrogen via the $\mathrm{a}^{\prime \prime}{ }^{1} \Sigma_{\mathrm{g}}^{+}$excited state as a representative example. Our results may be used to rationalize relative fs and hfs populations in molecular cations produced by REMPI in subsequent spectroscopy or dynamics experiments and may thus assist the interpretation of experimental data. Moreover, they may serve as a theoretical background to develop future fine- and hyperfine-stateselective production schemes for molecular cations.

\section{ACKNOWLEDGMENTS}

This work has been supported by the Swiss National Science Foundation as part of the National Centre of Competence in Research, Quantum Science and Technology (NCCR-QSIT), the European Commission under the Seventh Framework Programme No. FP7 GA 607491 COMIQ and the University of Basel.

${ }^{1}$ M. N. R. Ashfold and J. D. Howe, Annu. Rev. Phys. Chem. 45, 57 (1994).

${ }^{2}$ A. M. Ellis, M. Feher, and T. G. Wright, Electronic and Photoelectron Spectroscopy (Cambridge University Press, Cambridge, 2005).

${ }^{3}$ R. J. S. Morrison, W. E. Conaway, T. Ebata, and R. N. Zare, J. Chem. Phys. 84, 5527 (1986).

${ }^{4}$ R. D. Guettler, G. C. Jones, Jr., L. A. Posey, and R. N. Zare, Science 266, 259 (1994).

${ }^{5}$ X. Tong, T. Nagy, J. Yosa Reyes, M. Germann, M. Meuwly, and S. Willitsch, Chem. Phys. Lett. 547, 1 (2012).

${ }^{6} \mathrm{M}$. Germann, X. Tong, and S. Willitsch, Nat. Phys. 10, 820 (2014).

${ }^{7}$ S. R. Mackenzie, F. Merkt, E. J. Halse, and T. P. Softley, Mol. Phys. 86, 1283 (1995).

${ }^{8}$ X. Tong, A. H. Winney, and S. Willitsch, Phys. Rev. Lett. 105, 143001 (2010)

${ }^{9}$ X. Tong, D. Wild, and S. Willitsch, Phys. Rev. A 83, 023415 (2011).

${ }^{10}$ M. Germann and S. Willitsch, J. Chem. Phys. 145, 044314 (2016).

${ }^{11}$ S. W. Allendorf, D. J. Leahy, D. C. Jacobs, and R. N. Zare, J. Chem. Phys. 91, 2216 (1989).

${ }^{12}$ K. L. Reid, D. J. Leahy, and R. N. Zare, J. Chem. Phys. 95, 1746 (1991).

${ }^{13}$ R. G. Bray and R. M. Hochstrasser, Mol. Phys. 31, 1199 (1976).

${ }^{14}$ C. Maïnos, Phys. Rev. A 33, 3983 (1986).

${ }^{15}$ S. N. Dixit and V. McKoy, J. Chem. Phys. 82, 3546 (1985).

${ }^{16} \mathrm{H}$. Lefebvre-Brion and R. W. Field, The Spectra and Dynamics of Diatomic Molecules (Elsevier, Amsterdam, 2004).

${ }^{17}$ M. Hippler, Mol. Phys. 97, 105 (1999). 
${ }^{18}$ M. Göppert, Naturwissenschaften 17, 932 (1929).

${ }^{19}$ M. Göppert-Mayer, Ann. Phys. 401, 273 (1931).

${ }^{20}$ Although the overall [2+1'] REMPI scheme is a resonant process (in the sense that ionization is achieved via a neutral excited state), the twophoton, bound-bound transition connecting the neutral ground state to the neutral excited state regarded by itself is a non-resonant process.

${ }^{21}$ R. N. Zare, Angular Momentum (John Wiley \& Sons, New York, 1988).

${ }^{22}$ A. R. Edmonds, Drehimpulse in der Quantenmechanik (Bibliographisches Institut, Mannheim, 1964).

${ }^{23}$ M. Germann, Ph.D. thesis, University of Basel, 2016.

${ }^{24}$ T. F. Hanisco and A. C. Kummel, J. Phys. Chem. 95, 8565 (1991).

${ }^{25}$ For the signs of $\mu_{+} \mu_{-}^{\prime}$ and $\mu_{-}^{\prime \prime} \mu_{+}^{\prime \prime}$ different conventions are found in the literature. Here, the same sign convention as in Ref. 24 has been chosen, differing from the one used in Ref. 13.

${ }^{26}$ The quantity $S\left(M_{\mathrm{e}}\right)$ defined in Eq. (31) does not fully comply with the usual definition of a spectroscopic line strength, which involves sums over all degenerate states of the initial and the final level. $S\left(M_{\mathrm{e}}\right)$ is rather just a quantity proportional to the excitation rate populating a certain $\boldsymbol{M}_{\mathrm{e}}$-Zeeman state and hence to the population in this state after a given excitation period. Nonetheless, the symbol $S$ is used for this quantity here as well.

${ }^{27}$ The square root in the magnitude of the vibronic transition matrix element has been introduced in order that the relation $C_{k}$ $=\left|\left\langle n^{+} \Lambda^{+}, v^{+}\left|\mathrm{T}_{\Delta \Lambda}^{\prime k}\right| n^{\prime} \Lambda^{\prime}, v^{\prime}\right\rangle\right|^{2}$ still holds.

${ }^{28}$ The phases $\phi_{0}, \phi_{2}$ of the vibronic transition matrix elements can be related to the scattering phases of the photoelectron; see, e.g., Eqs. (9) and (10) in J. Xie and R. N. Zare, J. Chem. Phys. 97, 2891 (1992).

${ }^{29}$ F. Merkt and T. P. Softley, J. Chem. Phys. 96, 4149 (1992).

${ }^{30}$ G. Öhrwall, P. Baltzer, and J. Bozek, Phys. Rev. A 59, 1903 (1999).

${ }^{31}$ H. Palm and F. Merkt, Phys. Rev. Lett. 81, 1385 (1998).

${ }^{32}$ For both the excitation and the ionization step, the polarization of the radiation has been assumed parallel to the $z$-axis of the space-fixed frame.
Allowing for an angle between the two polarization vectors thus implies that the calculations for these two steps refer to two different space-fixed frames.

${ }^{33}$ J. M. Brown and A. Carrington, Rotational Spectroscopy of Diatomic Molecules (Cambridge University Press, Cambridge, 2003).

${ }^{34} \mathrm{P}$. R. Bunker and P. Jensen, Molecular Symmetry and Spectroscopy, 2nd ed. (NRC Research Press, Ottawa, 2006).

${ }^{35}$ C. Maïnos, Y. Le Duff, and E. Boursey, Mol. Phys. 56, 1165 (1985).

${ }^{36}$ C. Maïnos and Y. Le Duff, Mol. Phys. 60, 383 (1987).

${ }^{37}$ D. J. Leahy, K. L. Reid, and R. N. Zare, J. Chem. Phys. 95, 1757 (1991).

${ }^{38}$ R. S. Freund, T. A. Miller, D. De Santis, and A. Lurio, J. Chem. Phys. 53, 2290 (1970).

${ }^{39}$ D. De Santis, A. Lurio, T. A. Miller, and R. S. Freund, J. Chem. Phys. 58, 4625 (1973).

${ }^{40}$ A. C. Legon and P. W. Fowler, Z. Naturforsch. A 47, 367 (1992).

${ }^{41}$ N. Berrah Mansour, C. Kurtz, T. C. Steimle, G. L. Goodman, L. Young, T. J. Scholl, S. D. Rosner, and R. A. Holt, Phys. Rev. A 44, 4418 (1991).

${ }^{42}$ J. Mur-Petit, J. J. García-Ripoll, J. Pérez-Ríos, J. Campos-Martínez, M. I. Hernández, and S. Willitsch, Phys. Rev. A 85, 022308 (2012).

${ }^{43}$ F. Wolf, Y. Wan, J. C. Heip, F. Gebert, C. Shi, and P. O. Schmidt, Nature 530, 457 (2016).

${ }^{44}$ A. W. Kam and F. M. Pipkin, Phys. Rev. A 43, 3279 (1991).

${ }^{45}$ K. R. Lykke and B. D. Kay, J. Chem. Phys. 95, 2252 (1991).

${ }^{46}$ M. J. J. Vrakking, A. S. Bracker, and Y. T. Lee, J. Chem. Phys. 96, 7195 (1992).

${ }^{47}$ E. J. Salumbides, A. Khramov, and W. Ubachs, J. Phys. Chem. A 113, 2383 (2009).

${ }^{48}$ As before, mixing of Hund's case $\left(b_{\beta_{J}}\right)$ basis states has been neglected here for simplicity. Including this mixing has only a minor effect on the results shown (see Ref. 23). 